\title{
Evidence for powerful AGN winds at high redshift: dynamics of galactic outflows in radio galaxies during the "Quasar Era" *
}

\author{
N. P. H. Nesvadba ${ }^{1,2}$, M. D. Lehnert ${ }^{1}$, C. De Breuck ${ }^{3}$, A. M. Gilbert ${ }^{4}$, and W. van Breugel ${ }^{5}$
}

\author{
1 GEPI, Observatoire de Paris, CNRS, Université Denis Diderot, 5 place Jules Janssen, 92190 Meudon, France \\ e-mail: nicole.nesvadba@obspm.fr \\ 2 Marie-Curie Fellow \\ 3 European Southern Observatory, Karl-Schwarzschild Strasse, 85748 Garching bei München, Germany \\ 4 The Aerospace Corporation, PO Box 92957, MS M2-266, El Segundo, CA 90245, USA \\ 5 University of California, Merced, PO Box 2039, Merced, CA 95344, USA
}

Recieved 8 June 2008 / Accepted 30 August 2008

\section{ABSTRACT}

\begin{abstract}
AGN feedback now appears as an attractive mechanism to resolve some of the outstanding problems with the "standard" cosmological models, in particular those related to massive galaxies. At low redshift, evidence is growing that gas cooling and star formation may be efficiently suppressed by mechanical energy input from radio sources. To directly constrain how this may influence the formation of massive galaxies near the peak in the redshift distribution of powerful quasars, $z \sim 2$, we present an analysis of the emission-line kinematics of 3 powerful radio galaxies at $z \sim 2-3$ (HzRGs) based on rest-frame optical integral-field spectroscopy obtained with SINFONI on the VLT. The host galaxies of powerful radio-loud AGN are among the most massive galaxies, and thus AGN feedback may have a particularly clear signature in these galaxies.

We find evidence for bipolar outflows in all HzRGs, with kinetic energies that are equivalent to $0.2 \%$ of the rest-mass of the supermassive black hole. Observed total velocity offsets in the outflows are $\sim 800-1000 \mathrm{~km} \mathrm{~s}^{-1}$ between the blueshifted and redshifted line emission, and $F W H M \mathrm{~s} \sim 1000 \mathrm{~km} \mathrm{~s}^{-1}$ suggest strong turbulence. Line ratios allow to measure electron temperatures, $\sim 10^{4} \mathrm{~K}$ from [OIII] $] \lambda \lambda 4363,4959,5007$ at $z \sim 2$, electron densities $\left(\sim 500 \mathrm{~cm}^{-3}\right)$ and extinction $\left(A_{V} \sim 1-4 \mathrm{mag}\right)$. Ionized gas masses estimated from the $\mathrm{H} \alpha$ luminosity are of order $10^{10} M_{\odot}$, similar to the molecular gas content of HzRGs, underlining that these outflows may indicate a significant phase in the evolution of the host galaxy. The total energy release of $\sim 10^{60}$ erg during a dynamical time of $\sim 10^{7}$ yrs corresponds to about the binding energy of a massive galaxy, similar to the prescriptions adopted in galaxy evolution models. Geometry, timescales and energy injection rates of order $10 \%$ of the kinetic energy flux of the jet suggest that the outflows are most likely driven by the radio source. The global energy density release of $\sim 10^{57} \mathrm{erg} \mathrm{s}^{-1} \mathrm{Mpc}^{-3}$ may also influence the subsequent evolution of the HzRG by enhancing the entropy and pressure in the surrounding halo and facilitating ram-pressure stripping of gas in satellite galaxies that may contribute to the subsequent mass assembly of the HzRG through low-dissipation "dry" mergers.
\end{abstract}

Key words. galaxies: high-redshift - galaxies: formation - galaxies: jets - infrared: galaxies - galaxies: kinematics and dynamics

\section{Introduction}

AGN feedback has now become a crucial process in most models of galaxy formation and evolution. Current models postulate that massive galaxies experienced an early epoch of vigorous star formation that was terminated by a nearly instantaneous, powerful blow-out phase triggered by the AGN (e.g. Silk \& Rees 1998; Scannapieco \& Oh 2004; Springel et al. 2005; Di Matteo et al. 2005; Croton et al. 2006; Bower et al. 2006; Hopkins et al. 2006). Such a mechanism would heat and remove most of the ambient gas of the galaxy, and thus reconcile the discrepancy between the hierarchical standard model of galaxy formation and observational constraints.

Bolometric luminosities of powerful AGN at high redshift correspond to an energy output of $10^{60-61}$ erg during an AGN lifetime of $10^{7-8} \mathrm{yrs}$. Although this is equal, or even exceeds the binding energy of a massive galaxy, this energy will only have

* Based on observations collected at the European Southern Observatory, Very Large Telescope Array, Cerro Paranal, Chile (076.A-0684(A)). an impact on the evolution of the host galaxy, if it is efficiently transferred to the ambient gas with a coupling efficiency of at least a few percent.

Observationally, evidence is growing that AGN feedback may be related mostly to radio-loud AGN. Perhaps the most spectacular examples at low redshift are the giant cavities in the $\mathrm{X}$-ray halos of massive galaxy clusters, which appear to inject few $\times 10^{60}$ erg into the surrounding gas within a few $\times 10^{7}$ yrs, sufficient to suppress gas cooling (Boehringer et al. 1993; Bîrzan et al. 2004; Rafferty et al. 2006; McNamara \& Nulsen 2007). Best et al. $(2005,2006)$ find more subtle, yet general evidence that radio sources may balance gas cooling in $\sim 2000$ radio-loud early-type galaxies taken from the Sloan Digital Sky Survey and the NVSS and FIRST radio surveys. Very recently, Donoso et al. (2008) found that the fraction of radio-loud galaxies as a function of stellar mass increases with increasing redshift, in particular for massive galaxies. Interestingly, the fraction of radio-loud galaxies seems to be a strong function of mass, suggesting that such radio-driven feedback will act predominantly in the most massive galaxies, in broad agreement with the 
predictions of galaxy evolution models. This is also supported by the observation that powerful radio galaxies are among the most massive galaxies at all redshifts (De Breuck et al. 2001; Willott et al. 2003; Rocca-Volmerange et al. 2004). Since AGN feedback is expected to act predominantly in massive galaxies, we may expect that its influence will be most clearly expressed in particularly massive galaxies.

However, since massive early-type galaxies appear to have completed most of their growth at high redshift (e.g., Rudnick et al. 2003, and references therein), directly investigating the impact of jet-driven AGN feedback during the formation of massive galaxies requires direct observations of radio-loud galaxies at $z \geq 1$. Interestingly, longslit spectroscopy of powerful radio galaxies at $z \geq 1$ (and in a few cases at lower redshifts) revealed strongly distorted emission line kinematics, with velocities often exceeding $\sim 1000 \mathrm{~km} \mathrm{~s}^{-1}$ along the axis of the radio jet (e.g., Tadhunter 1991; McCarthy et al. 1996; Evans 1998; Baum \& McCarthy 2000; Villar-Martín et al. 1999; Inskip et al. 2002; Clark et al. 1998; Best et al. 1997). Similarly, [OIII] $\lambda 5007$ narrow-band imaging of 4C 19.71 at $z \sim 3.6$ revealed a giant emission line region extending over $\sim 74 \mathrm{kpc}$ (Armus et al. 1998). This emission line region, corresponding to an ionized gas mass of $10^{8-9} M_{\odot}$ aligns roughly with the axis of the radio jet, which led Armus et al. to suspect that the jet and the nebulosity may be physically related. Heckman et al. (1991b,a) also find this for radio-loud quasars. Moreover, Ly $\alpha$ spectroscopy and line imaging revealed luminous emission line halos with sizes that appear related to the size of the radio jet, sometimes with kinematically more quiescent gas beyond (e.g. Villar-Martín et al. 2003, 2006, 2007).

However, the complexity of the velocity fields and relatively large spatial extent of the emission line regions made it difficult to robustly infer the global characteristics of these nebulae. Integral-field spectrographs now open a new avenue to study the underlying dynamical mechanisms with unprecedented robustness and across the full two-dimensional surface of the emission line nebulae. Over recent years, our understanding of galaxy evolution in the early universe has significantly advanced due to the discovery of large numbers of galaxies at similar redshifts (e.g., Steidel et al. 1996; Smail et al. 2002), and during this time, modeling of galaxy and structure formation has improved. This allows us to reassess the emission line kinematics of powerful high-redshift radio galaxies (HzRGs). Near-infrared studies are particularly well suited to study the inner regions of HzRG extended emission line regions, allowing for relatively high spatial resolution of $\sim 0.5^{\prime \prime}$ even with seeing-limited observations, and opening a window to the rest-frame optical line emission where extinction is less severe than in the rest-frame UV.

Nesvadba et al. (2006b) find strong evidence for energetic outflows of ionized gas from rest-frame optical integral-field spectroscopy of the powerful radio galaxy MRC1138-262 at $z \sim 2.2$. With a spatial extent of $30 \mathrm{kpc}$, relative velocities and line widths of $F W H M \sim 1000 \mathrm{~km} \mathrm{~s}^{-1}$ across the source, corresponding to kinetic energies of few $\times 10^{60} \mathrm{erg}$, this gas may be experiencing a feedback episode as dramatic as necessary to unbind a significant fraction of the ambient gas from the halo of a massive galaxy. The kinetic energy of the outflow corresponds to a few percent of the jet kinetic energy, and the dynamical timescale of a few $\times 10^{7}$ yrs appears roughly similar to the age of the radio source. Moreover, the turbulent, luminous gas extends to the size of the radio jets. In contrast, very similar observations do not show evidence for such outflows in compact radio galaxies at similar redshifts (Nesvadba et al. 2007a), which are likely the younger analogs to extended HzRGs. This agrees with expectations. If the entrainment rates are similar, then only a few percent of the ISM will already be affected by the radio source for ages less than $\sim 10^{6}$ yrs.

Currently, the host galaxies of powerful, radio-loud AGN (HzRGs and quasars) represent the only galaxy population at $z \geq$ 2 with such extreme kinematics. To address the question whether MRC1138-262 is a "one-off", or whether AGN feedback is a common phenomenon in HzRGs, we continue the analysis of Nesvadba et al. (2006b, 2007a), adding another three HzRGs with near-infrared integral-field data at $z \approx 2-3$. Comparing with the samples of Iwamuro et al. (e.g., 2003); De Breuck et al. (e.g., 2000); Humphrey et al. (e.g., 2008); Baum \& McCarthy (e.g., 2000), we find that their rest-frame UV-optical properties are within the typical range of powerful HzRGs. These galaxies have extended radio morphologies, and relatively similar radio power to MRC1138-262, $\mathcal{P}_{1.4 \mathrm{GHz}} \sim 10^{26} \mathrm{~W} \mathrm{~Hz}^{-1}$. Thus, they are among the most powerful radio galaxies observed at all redshifts. Given the observational difficulties in studying highredshift galaxies, this seems the most promising way of identifying the fingerprints of powerful AGN-driven feedback and underlying physical processes directly from the gas kinematics of powerful AGN host galaxies at $z \sim 2$.

However, these radio powers are not exceptional in the early universe. Willott et al. (2001) studied the redshift evolution of the radio luminosity function and find a population of galaxies with radio powers $\mathcal{P}_{151 \mathrm{MHz}} \sim 10^{26.5-29.5} \mathrm{~W} \mathrm{~Hz}^{-1}$ at $151 \mathrm{MHz}$, which shows a strong peak near $z \sim 2$ and rapidly declines towards lower redshifts. Finally, the radio emissivity is likely a strong function of environment and evolutionary stage of the synchrotron plasma (e.g., Kaiser et al. 1997a,b), causing changes in the radio luminosity of more than an order of magnitude. Hence, by selecting particularly powerful sources, we may bias our sample more in terms of the evolutionary stage or environment than the kinetic luminosity of the radio source.

Specifically, we observed MRC0316-257 at $z=3.13$, MRC0406-244 at $z=2.42$, and TXS0828+192 at $z=2.57$. Entrained ionized gas masses and kinetic energies seem very similar to MRC1138-262 in all three cases. This leads us to propose that AGN feedback may be a common phenomenon in powerful radio galaxies during the "Quasar Era".

\section{Observations and data reduction}

Observations in the $H$ and $K$ bands were carried out with the integral-field spectrograph SINFONI (Bonnet et al. 2004) on the VLT in December 2005 under good conditions.

SINFONI is a medium-resolution, image-slicing integralfield spectrograph, with $8^{\prime \prime} \times 8^{\prime \prime}$ field of view at a $0.25^{\prime \prime} \times$ $0.25^{\prime \prime}$ pixel scale, and spectral resolution of $R \sim 3000$, and $R \sim 4000$ in the $H$, and $K$ bands, respectively. Individual exposure times were $450 \mathrm{~s}$ in $H$ and $600 \mathrm{~s}$ in $K$, respectively. Total exposure times are given for each band and each source in Table 1.

We use the IRAF (Tody 1993) standard tools for the reduction of longslit-spectra, modified to meet the special requirements of integral-field spectroscopy, and complemented by a dedicated set of IDL routines. Data are dark-frame subtracted and flat-fielded. The position of each slitlet is measured from a set of standard SINFONI calibration data, measuring the position of an artificial point source. Rectification along the spectral axis and wavelength calibration are done before night sky subtraction to account for some spectral flexure between the frames. Curvature is measured and removed using an arc lamp, before shifting the spectra to an absolute (vacuum) wavelength scale 
Table 1. Sample and observation log.

\begin{tabular}{lcccc}
\hline \hline $\begin{array}{l}\text { Source } \\
(1)\end{array}$ & $\begin{array}{c}z \\
(2)\end{array}$ & $\begin{array}{c}\text { Filter } \\
(3)\end{array}$ & $\begin{array}{c}\text { ToT } \\
(4)\end{array}$ & $\begin{array}{c}\text { Seeing } \\
(5)\end{array}$ \\
\hline $0316-257$ & 3.13 & $K$ & 100 & $0.7^{\prime \prime} \times 0.4^{\prime \prime}$ \\
\hline $0406-244$ & 2.44 & $H$ & 90 & $0.7^{\prime \prime} \times 0.6^{\prime \prime}$ \\
$0406-244$ & & $K$ & 100 & $0.8^{\prime \prime} \times 0.6^{\prime \prime}$ \\
\hline $0828+193$ & 2.57 & $H$ & 135 & $0.7^{\prime \prime} \times 0.5^{\prime \prime}$ \\
$0828+193$ & & $K$ & 170 & $0.7^{\prime \prime} \times 0.5^{\prime \prime}$ \\
\hline
\end{tabular}

Column (1) - source ID. Column (2) - redshift. Column (3) - total exposure time (on target) in min. Column (4) - seeing. For TXS0828+193, the seeing was determined from a star within the field of view. Otherwise, we estimated the seeing from the standard star.

with reference to the $\mathrm{OH}$ lines in the data. To account for variations in the night sky emission, we normalize the sky frame to the average of the object frame separately for each wavelength before sky subtraction, masking bright foreground objects, and correcting for residuals of the background subtraction and uncertainties in the flux calibration by subsequently subtracting the (empty sky) background separately from each wavelength plane. In the $H$ band, where the suppression of night sky lines is particularly difficult, we additionally clipped all strongly deviant pixels at wavelengths near prominent night sky lines.

The three-dimensional data are then reconstructed and spatially aligned using the telescope offsets as recorded in the header within the same observing block (a sequence of typically 5 to 10 individual exposures with a total length of about an hour), and by cross-correlating the line images from the combined data in each observing block, to eliminate relative offsets between different observing blocks. Telluric correction is applied to each individual cube before the cube combination. Flux scales are obtained from standard star observations. From the light profile of the standard star, we measure the $F W H M$ spatial resolution in the combined cube, which is typically $\sim 0.6^{\prime \prime}$.

\subsection{Complementary data sets and alignment}

We complement our SINFONI data through deep, A-array 1.4 GHz radio maps of Carilli et al. (1997) with a spatial resolution of $\sim 0.4^{\prime \prime}$. These data were kindly provided to us by Carilli. Studying the interplay between radio jets and the ISM of high-redshift galaxies requires a relative alignment that is accurate to $\leq 0.5^{\prime \prime}$, which is better than the accuracy of the absolute astrometry reached with the VLT. We therefore assume that the radio core falls onto the center of the continuum emission for all sources. Note that this coincides with the position of the largest velocity gradient in the velocity maps of TXS0828+193 and MRC0406-244 to within less than a few tenths of a second of arc. Remaining small offsets are likely due to projection effects. Since we did not detect MRC0316-257 in the continuum, we align the radio core with the steepest gradient in the velocity map, implying that the basic physical mechanism of the outflow is similar in the 3 sources - which is justified given their overall similarity. We also note that, unlike in MRC1138-262 (Nesvadba et al. 2006b), we do not find a nuclear broad-line region related to the AGN in any of the three sources.

\section{Continuum and line emission morphologies and integrated spectra}

We constructed continuum-free line images by summing over the spectral regions of the emission lines in the integrated

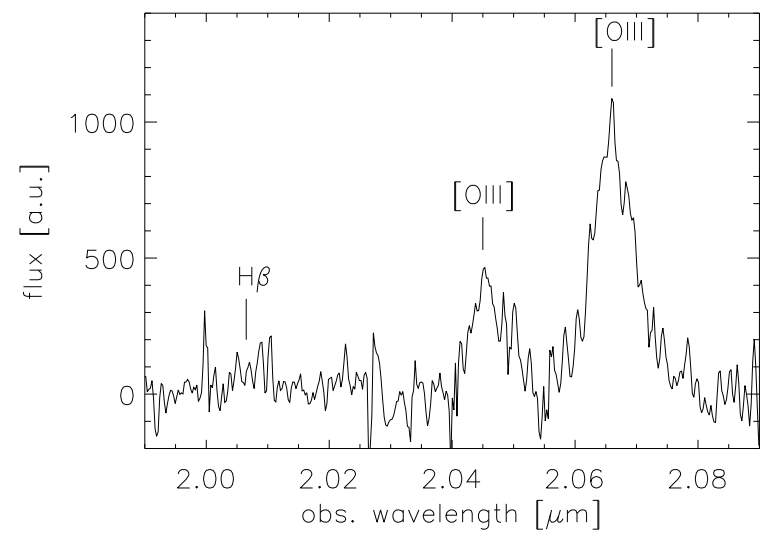

Fig. 1. Integrated $K$ band spectrum of MRC0316-257 at $z=3.13$, extracted from all pixels in which the [OIII] $\lambda 5007$ line emission exceeded $3 \sigma$, and covering the $[\mathrm{OIII}] \lambda \lambda 4959,5007$ doublet and $\mathrm{H} \beta$.

spectra, and removing the underlying continuum emission. Rather than adopting a fixed aperture, we selected all pixels where the [OIII] $\lambda 5007$ emission line was detected at a signal-tonoise ratio greater than 3 . This helps to maximize the signal-tonoise ratio, which may otherwise be affected by night-sky residuals, whose strength often varies across the detector. To extract line-free continuum images, we fitted the spectrum of each spatial pixel where it is unaffected by bright emission lines or strong night sky line residuals. We interpolated over the contaminated parts before collapsing the spectra.

\section{1. $M R C 0316-257$}

The integrated spectrum of MRC0316-257 is shown in Fig. 1. The [OIII] $\lambda \lambda 44959,5007$ doublet and $\mathrm{H} \beta$ are detected at redshifts of $z=3.1273^{1}$ and $z=3.1292$ for [OIII] $\lambda 5007$, respectively, and have line widths of $F W H M=1465 \mathrm{~km} \mathrm{~s}^{-1}$ and $F W H M=900 \mathrm{~km} \mathrm{~s}^{-1}$ for $[\mathrm{OIII}] \lambda 5007$ and $\mathrm{H} \beta$, respectively ${ }^{2}$. See Table 2 for a summary of the emission line properties and related uncertainties.

We show the $[\mathrm{OIII}] \lambda 5007$ emission line morphology in Fig. 8. Line emission is detected over an area of $\approx 2.8^{\prime \prime} \times 1.2^{\prime \prime}$ $(21 \mathrm{kpc} \times 9 \mathrm{kpc})$, and is elongated along the axis of the radio jet (the 1.4 GHz VLA map is shown as contours in Fig. 8). $\mathrm{H} \beta$ and continuum emission are very faint, so that their morphology could not be measured.

\section{2. $M R C 0406-244$}

MRC0406-244 at $z=2.44^{3}$ has a splendid hour-glass shaped, rest-frame UV and optical morphology which has previously been studied with NICMOS and WFPC imaging on-board the Hubble Space Telescope (Pentericci et al. 1999), as well as with ground-based observations (McCarthy et al. 1991, 1992; Rush et al. 1997) in the optical and near-infrared. As pointed out by Rush et al. (1997), the morphology is a strong function of

\footnotetext{
1 Adopting the flat $\Omega_{\Lambda}=0.7$ cosmology with $H_{0}=70 \mathrm{~km} \mathrm{~s}^{-1} \mathrm{Mpc}^{-1}$ we use a luminosity distance $D_{\mathrm{L}}=26.7 \mathrm{Gpc}$ and angular size distance $D_{\mathrm{A}}=1.5 \mathrm{Gpc}$. The size scale is $7.6 \mathrm{kpc} /{ }^{\prime \prime}$. The age of the universe at $z \sim 3.13$ for this cosmological model is $2.0 \mathrm{Gyr}$.

2 Unless stated explicitly, all kinematic parameters are in the restframe.

3 The luminosity distance is $D_{\mathrm{L}}=19.8 \mathrm{Gpc}$ and angular size distance $D_{\mathrm{A}}=1.7 \mathrm{Gpc}$. The size scale is $8.1 \mathrm{kpc} /{ }^{\prime \prime}$. The age of the universe for this redshift and cosmological model is $2.6 \mathrm{Gyr}$.
} 
Table 2. Emission lines in MRC0316-257.

\begin{tabular}{lccccc}
\hline \hline $\begin{array}{l}\text { Line } \\
(1)\end{array}$ & $\lambda_{0}$ & $\lambda_{\text {obs }}$ & $z$ & FWHM & Flux \\
$(2)$ & $(3)$ & $(4)$ & $(5)$ & $(6)$ \\
\hline$[\mathrm{OIII}]$ & 5007 & $20665.4 \pm 4.2$ & $3.1273 \pm 0.0008$ & $1465 \pm 90$ & $2.2 \pm 0.1$ \\
{$[\mathrm{OIII}]$} & 4959 & $20459.6 \pm 4.3$ & $3.1257 \pm 0.0009$ & $1357 \pm 94$ & $0.9 \pm 0.1$ \\
$\mathrm{H} \beta$ & 4861 & $20072.2 \pm 8.1$ & $3.1292 \pm 0.0017$ & $902 \pm 266$ & $1.2 \pm 0.4$ \\
\hline
\end{tabular}

Column (1) - line. Column (2) - rest-frame wavelength in $\AA$. Column (3) - observed wavelength in Å. Column (4) - redshift. Column (5) rest-frame $F W H M$, corrected for instrumental resolution in $\mathrm{km} \mathrm{s}^{-1}$. Column (6) - line flux in units of $10^{-15} \mathrm{erg} \mathrm{s}^{-1} \mathrm{~cm}^{-2}$.

waveband. In particular, the $J$-band morphology is much more compact than, e.g., the morphology in the $K$ s or $g$-band. Rush et al. (1997) suspected that this is most likely due to contamination with strong emission lines ( $\mathrm{H} \alpha$ in the $K$ s band, Ly $\alpha$ in the $g$ band).

Our SINFONI data illustrate that this is indeed the case. In Fig. 9 we show the [OIII] $\lambda 5007$ emission line morphology. Contours indicate the line-free $K$-band continuum. Line emission is clearly extended over $3.7^{\prime \prime} \times 1.1^{\prime \prime}(30.1 \mathrm{kpc} \times$ $9.3 \mathrm{kpc}$ ) and is reminiscent of two edge-brightened bubbles with several brighter knots superimposed. HST NICMOS imaging of Pentericci et al. (1999) in the Fl60W filter (which covers [OIII] $\lambda 5007$ and is dominated by line emission) reveals a more complex morphology, with several bright knots and fainter filaments inbetween.

The line-free continuum morphology extracted from the SINFONI data cube shows that continuum emission is faint and relatively compact, but spatially resolved (contours in Fig. 9). We detect the continuum over an area of approximately $1.3^{\prime \prime} \times$ $0.6^{\prime \prime}(10.6 \mathrm{kpc} \times 5 \mathrm{kpc})$, corresponding to an integrated magnitude of $K=17.7 \mathrm{mag}$ in a $3^{\prime \prime}$ aperture centered on the continuum peak (compared to $K=17.5 \mathrm{mag}$ if not correcting for the line contamination). In the $H$ band, we measure $H=18.9$ mag and $H=19.3$ mag for the uncorrected and corrected magnitude, respectively. We also estimate an azimuthally-averaged half-light radius, which corresponds to the area of brightest continuum emission, that contains half the total continuum flux above a $3 \sigma$ threshold. We find a half-light radius of $r_{1 / 2} \sim 0.6^{\prime \prime}(\sim 5 \mathrm{kpc})$.

The integrated spectra of MRC0406-244 in the $H$ and $K$ bands are shown in Fig. 2. Rest-frame optical emission lines are very bright, in particular the [OIII] $\lambda \lambda 4959,5007$ doublet and $\mathrm{H} \beta$ in the $H$ band, and the blended $\mathrm{H} \alpha$ and [NII] $\lambda 6583$ emission lines in the $K$ band. In the $K$ band, we also detect the [SII] 26716 , 6731 doublet and [OI] $\lambda 6300$. Moreover, we marginally detect the faint HeII $\lambda 4686$ in the $H$ band spectrum. The inset in the left panel of Fig. 2 shows the fainter lines in the $H$-band. The profiles of some lines are affected by residuals of night-sky lines (e.g., on the red wing of $\mathrm{H} \beta$ ). See Table 3 for the observed wavelengths, redshifts, line widths and fluxes of the detected emission lines in the integrated spectrum.

\section{3. $T X S 0828+193$}

TXS0828+193 at $z=2.57^{4}$ is the galaxy with the most extended radio lobes in our sample (103 kpc for our cosmology). F675W WFPC2 imaging indicates a clumpy, irregular and asymmetric morphology at rest-frame UV wavelengths (Pentericci et al. 1999).

${ }_{4}$ The luminosity distance is $D_{\mathrm{L}}=21.1 \mathrm{Gpc}$ and angular size distance $D_{\mathrm{A}}=1.7 \mathrm{Gpc}$. The size scale is $8.0 \mathrm{kpc} /{ }^{\prime \prime}$. The age of the universe for this redshift and cosmological model is $2.5 \mathrm{Gyr}$.
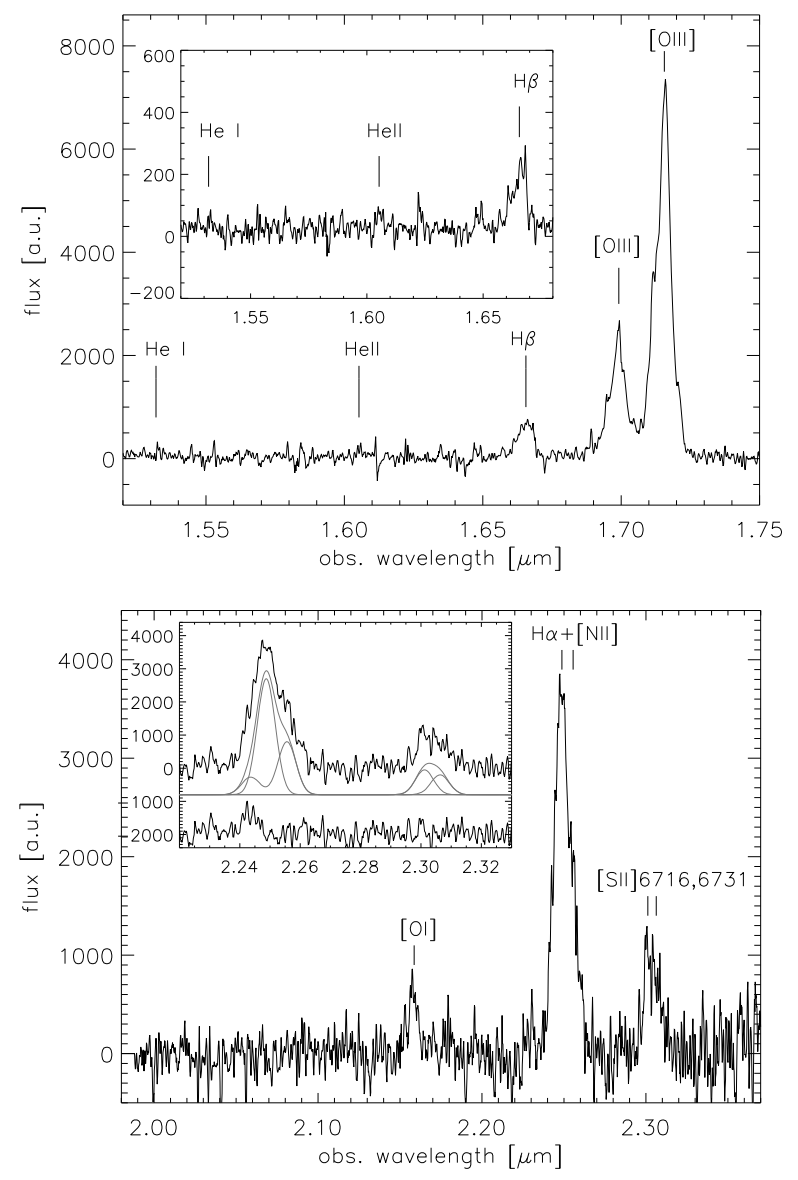

Fig. 2. Integrated $H$ (top) and $K$ band spectrum (bottom) of MRC0406244 at $z=2.42$, extracted from all pixels in which the [OIII] $\lambda 5007$ line emission exceeds $3 \sigma$. The inset in the upper panel shows the detected, relatively faint $\mathrm{H} \beta$, and $[\mathrm{HeII}] \lambda 4685$ lines, and the expected wavelength of [HeI] $\lambda 4471$, which is not detected. The inset in the right panel shows (from top to bottom): (1) the blended $\mathrm{H} \alpha$ and [NII] $\lambda \lambda 6548,6583$ lines, and the $[\mathrm{SII}] \lambda \lambda 6716,6731$ doublet (2) the best Gaussian fit to these lines (see text) (3) fit residuals. The fitted lines and residuals are shifted along the ordinate by arbitrary amouts.

TXS0828+193 is a particularly luminous line emitter. In Fig. 3 we show the integrated spectrum of TXS0828+193 in the $H$ and $K$ bands. [OIII] $\lambda \lambda 4959,5007, \mathrm{H} \beta, \mathrm{H} \gamma$ are detected in the $H$ band. [OIII] $\lambda 4363$ is faint, but detected with a significance of $3.6 \sigma$. In the $K$ band we observe [OI] $\lambda 6300, \mathrm{H} \alpha$ and [NII] $\lambda 6583$. The [SII] $\lambda \lambda 6716,6731$ doublet falls at observed wavelengths $\lambda>2.4$, which are dominated by telluric absorption. The emission line properties of the integrated spectrum of TXS0828+193 are summarized in Table 4.

We show the $[\mathrm{OIII}] \lambda 5007$ emission line morphology of TXS0828+193 in Fig. 10. The bright extended emission line regions are significantly more extended than the continuum 
Table 3. Emission lines in MRC0406-244.

\begin{tabular}{lccccc}
\hline \hline $\begin{array}{l}\text { Line } \\
(1)\end{array}$ & $\lambda_{0}$ & $\lambda_{\text {obs }}$ & $z$ & $F W H M$ & Flux \\
& $(2)$ & $(3)$ & $(4)$ & $(5)$ & $(6)$ \\
\hline $\mathrm{H} \beta$ & 4861 & $1.66532 \pm 0.00034$ & $2.42587 \pm 0.00071$ & $1250 \pm 83$ & $1.9 \pm 0.1$ \\
{$[\mathrm{OIII}]$} & 4959 & $1.69860 \pm 0.00034$ & $2.42528 \pm 0.00069$ & $1524 \pm 93$ & $2.2 \pm 0.1$ \\
{$[\mathrm{OIII}]$} & 5007 & $1.71495 \pm 0.00034$ & $2.42510 \pm 0.00068$ & $1349 \pm 81$ & $6.6 \pm 0.4$ \\
\hline$[\mathrm{OI}]$ & 6300 & $2.15777 \pm 0.00045$ & $2.42503 \pm 0.00072$ & $826 \pm 65$ & $0.74 \pm 0.07$ \\
$\mathrm{H} \alpha+[\mathrm{NII}]$ & 6563,6583 & $2.24879 \pm 0.00045$ & $2.42647 \pm 0.00060$ & $1756 \pm 106$ & $9.75 \pm 0.59$ \\
{$[\mathrm{SII}]$} & 6716,6731 & $2.30292 \pm 0.00048$ & $2.42492 \pm 0.00071$ & $1590 \pm 102$ & $2.40 \pm 0.16$ \\
\hline
\end{tabular}

Column (1) - line. Column (2) - rest-frame wavelength in $\AA$. Column (3) - observed wavelength in $\AA$. Column (4) - redshift. Column (5) rest-frame $F W H M$, corrected for instrumental resolution in $\mathrm{km} \mathrm{s}^{-1}$. Column (6) - line flux in units of $10^{-15} \mathrm{erg} \mathrm{s}^{-1} \mathrm{~cm}^{-2}$. For $\mathrm{H} \alpha$, [NII] $\lambda \lambda 6548$, 6583 and $[\mathrm{NII}] \lambda \lambda 6716,6731$ we give the values for the blended lines, respectively.
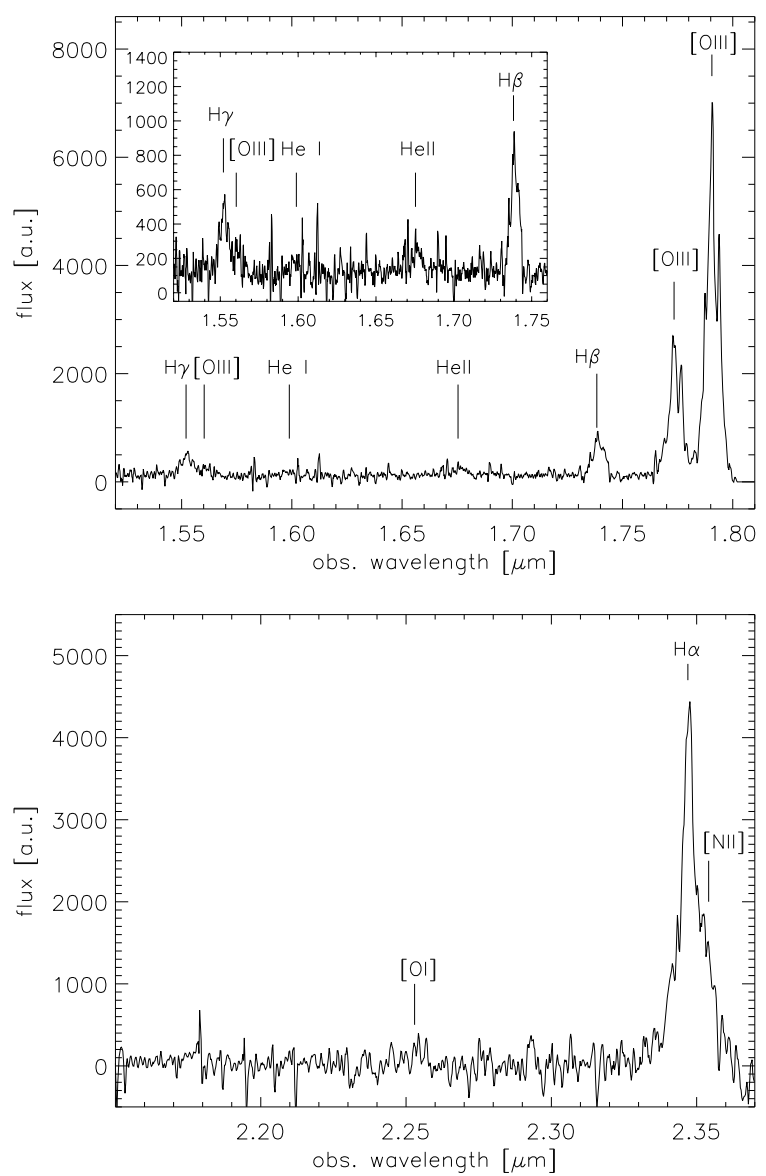

Fig. 3. Integrated $H$ (top) and $K$ band spectrum (bottom) of TXS0828+193 at $z=2.57$, extracted from all pixels in which the [OIII] $\lambda 5007$ line emission exceeds $3 \sigma$. The inset in the upper panel shows the detected, relatively faint $\mathrm{H} \beta, \mathrm{H} \gamma$, and [HeII] $\lambda 4685$ lines, and the expected wavelength of $[\mathrm{HeI}] \lambda 4471$, which is not detected. $[\mathrm{OIII}] \lambda \lambda 4959,5007$ have complex profiles, where the different components arise from the blue and redshifted bubble, respectively. [OIII] $\lambda 6300$ is faint, but detected with a significance of $5 \sigma$. In the $K$ band, we detect $\mathrm{H} \alpha$ and $[\mathrm{NII}] \lambda \lambda 6548,6583$, and the fainter $[\mathrm{OI}] \lambda 6300$ line. The [SII] $\lambda \lambda 6716,6731$ doublet is at observed wavelengths $>2.4 \mu \mathrm{m}$, which are dominated by telluric absorption.

(shown as contours in the left panel of Fig. 10), covering an area of $3.5^{\prime \prime} \times 1.2^{\prime \prime}(28 \mathrm{kpc} \times 9.6 \mathrm{kpc})$. The line image is dominated by two bright regions, an unresolved peak to the south, and an extended, more diffuse emission line region to the north-east. Comparison with the WFPC2 F675W image of Pentericci et al. (1999, their Fig. 7) indicates that the [OIII] $\lambda 5007$ emission line morphology and the rest-frame UV morphology are very similar.
The continuum is faint in the $H$ and $K$ band (approximately corresponding to the rest-frame $V$ and $R$ bands) and significantly more compact than the line emission. We show the line-free, combined $H$ and $K$ band continuum morphology as contours in Fig. 10. Continuum emission is detected over an area of $\sim 1.4^{\prime \prime} \times$ $0.8^{\prime \prime}$, corresponding to about $11.3 \mathrm{kpc} \times 6.4 \mathrm{kpc}$. Estimating an azimuthally-averaged half-light radius similar to Sect. 3.2, we find $0.47^{\prime \prime}(3.8 \mathrm{kpc})$ region. We estimate integrated magnitudes in the $H$ and $K$ band using $3^{\prime \prime}$ apertures, $K=19.4 \mathrm{mag}, H=$ $19.7 \mathrm{mag}$ (compared to $K=18.5 \mathrm{mag}$, and $H=19.1 \mathrm{mag}$, without correcting for line contamination).

\section{Characteristics of the extended emission line regions}

\subsection{Kinematics}

We extracted spectra from $3 \times 3$ pixel square apertures across the sources $\left(0.375^{\prime \prime} \times 0.375^{\prime \prime}\right)$ and fitted Gaussian line profiles to each spectrum. Relative velocities were estimated from the measured wavelengths of the line cores at each position (core of the strongest component in case of complex line profiles).

The large line widths observed in the extended emission line regions of HzRGs complicate the analysis of the spectra due to the blending of emission lines, in particular of $\mathrm{H} \alpha$ and [NII] $\lambda 6548,6583$. In order to obtain a consistent fit of all lines within each spectrum, we therefore estimated the redshift and line width from the [OIII] $\lambda \lambda 4959,5007$ doublet (whose components are luminous and can be isolated relatively easily) and imposed the same redshift and line width on all other emission lines. As a result, the line fluxes are the only free parameter in the fits to all other lines except [OIII].

However, such an approach implies that all emission lines trace the same gas kinematics. We tested this explicitly by comparing velocities and line widths obtained from [OIII] $\lambda \lambda 4959,5007$ in TXS0828+193 with those measured from $\mathrm{H} \beta$, in areas where $\mathrm{H} \beta$ is detected at a significant level $(>3 \sigma)$, and find good agreement between the measured velocities and line widths.

For much of the extended emission, the residuals suggest that fitting a single Gaussian component is adequate. However, in the central regions in particular, we do observe more complicated profiles and line splitting (see, e.g., Fig. 7). In these cases, we fitted 2-3 components with redshifts and line widths determined from the [OIII] $\lambda \lambda 4959,5007$ doublet, and leaving the flux of each line and each component as a free parameter.

We present the maps of rest-frame relative velocities and line widths (full width at half maximum) of the 3 galaxies in Figs. 8 to 10 . Overall, the velocity fields are remarkably similar 
Table 4. Emission lines in TXS0828+193.

\begin{tabular}{lccccc}
\hline \hline $\begin{array}{l}\text { Line } \\
(1)\end{array}$ & $\lambda_{0}$ & $\begin{array}{c}\lambda_{\text {obs }} \\
(3)\end{array}$ & $\begin{array}{c}z \\
(4)\end{array}$ & $\begin{array}{c}F W H M \\
(5)\end{array}$ & $\begin{array}{c}\text { Flux } \\
(6)\end{array}$ \\
\hline $\mathrm{H} \beta$ & 4861 & $1.73883 \pm 0.00039$ & $2.57710 \pm 0.0008$ & $1448 \pm 112$ & $0.6 \pm 0.0540902$ \\
{$[\mathrm{OIII}]$} & 4959 & $1.77422 \pm 0.00036$ & $2.57778 \pm 0.0007$ & $1239 \pm 76$ & $2.1 \pm 0.132873$ \\
{$[\mathrm{OIII}]$} & 5007 & $1.79112 \pm 0.00036$ & $2.57723 \pm 0.0007$ & $1185 \pm 71$ & $5.9 \pm 0.357164$ \\
\hline$[\mathrm{OI}]$ & 6300 & $2.2547 \pm 0.0006$ & $2.57821 \pm 0.0009$ & $1320 \pm 132$ & $0.5 \pm 0.1$ \\
$\mathrm{H} \alpha+[\mathrm{NII}]$ & 6563,6583 & $2.34705 \pm 0.00047$ & $2.57619 \pm 0.0007$ & $1176 \pm 47$ & $5.1 \pm 0.3$ \\
\hline
\end{tabular}

Column (1) - line. Column (2) - rest-frame wavelength in $\AA$. Column (3) - observed wavelength in Å. Column (4) - redshift. Column (5) rest-frame $F W H M$, corrected for instrumental resolution in $\mathrm{km} \mathrm{s}^{-1}$. Column (6) - line flux in units of $10^{-15} \mathrm{erg} \mathrm{s}^{-1} \mathrm{~cm}^{-2}$. For $\mathrm{H} \alpha$, [NII] $\lambda \lambda 6548$, 6583 and $[\mathrm{NII}] \lambda \lambda 6716,6731$ we give the values for the blended lines, respectively.

in all targets. In each galaxy we identify two regions with relatively homogeneous internal kinematics, which extend from near the radio core, and projected velocities relative to each other of $\sim 700-1000 \mathrm{~km} \mathrm{~s}^{-1}$. Velocities change abruptly in the central region of the velocity field, within about a spatial resolution element of our data sets, corresponding to $\sim 0.6^{\prime \prime}$ or $\leq 5 \mathrm{kpc}$. The velocity gradients for the individual galaxies are given in Table 6.

The velocity maps show substructure within each region, although the velocity gradients are significantly smaller than the large velocity shift near the center of the galaxies. Velocity gradients amount to $\sim 100-200 \mathrm{~km} \mathrm{~s}^{-1}$. We emphasize that the three targets have similar velocity fields, in spite of different morphologies.

Figures 8 to 10 also show maps of the $F W H M$ line widths. Overall, line widths are $F W H M \sim 500-1200 \mathrm{~km} \mathrm{~s}^{-1}$ in the three galaxies. Note that much of this range of FWHMs is due to variations in the line widths within individual areas, and not so much due to large variation between galaxies in our sample.

Apparent peaks in the central regions with $F W H M \geq$ $1400 \mathrm{~km} \mathrm{~s}^{-1}$ are likely artefacts due to the large velocity gradients and partial overlap of line emission from the two sides within one seeing disk. We also observe line splitting (see Fig. 7) in this area, and the observed line widths are consistent with what would be expected from blended lines with the observed line widths and velocity gradient in and between the two regions. In other regions of the galaxies, the emission line widths likely reflect a large intrinsic turbulent motion, since the widths exceed the velocity gradients within each bubble (at least on kpc scales).

\subsection{Extinction}

MRC0406-244 and TXS0828+193 are bright $\mathrm{H} \alpha$ and $\mathrm{H} \beta$ emitters and allow to construct maps of the $\mathrm{H} \beta / \mathrm{H} \alpha$ line ratios and thus extinction. For MRC0316-257 this is not possible, due to the larger redshift, $z=3.13$, at which $\mathrm{H} \alpha$ cannot be observed from the ground, and because $\mathrm{H} \beta$ is relatively faint.

We measured the $\mathrm{H} \beta / \mathrm{H} \alpha$ line ratios from $0.375^{\prime \prime} \times$ $0.375^{\prime \prime}$ box apertures $(3 \times 3$ pixels $)$. To derive extinctions, we use a galactic extinction law and an intrinsic (unreddened) Balmer decrement $\mathrm{H} \alpha / \mathrm{H} \beta=2.9$. (See Sect. 4.1 for a description of how we correct for blending between $\mathrm{H} \alpha$ and the [NII] $\lambda \lambda 6548,6583$ doublet.)

We show the extinction maps of MRC0406-244 and TXS0828+193 in the top and bottom panel of Fig. 4, respectively. Contours indicate the [OIII] $\lambda 5007$ emission line morphology to ease orientation. Extinctions at the wavelength of $\mathrm{H} \beta$, $A(\mathrm{H} \beta)$ vary between $A(\mathrm{H} \beta) \sim 1-4.5 \mathrm{mag}$ in both galaxies. (We give $A(\mathrm{H} \beta)=A(V)+0.14 \mathrm{mag}$, because we measured the extinction from the emission lines.) The most heavily extincted regions are near the center of each source, where $A(\mathrm{H} \beta) \sim 4-4.5$; the spatial extent of these areas corresponds approximately to the size of the continuum emission. Extinction in the extended emission line regions is less severe, with $A(\mathrm{H} \beta)_{\mathrm{ext}}^{0406} \sim 1-2 \mathrm{mag}$ in MRC0406-244, and $A(\mathrm{H} \beta)_{\mathrm{ext}}^{0828} \sim 2-3 \mathrm{mag}$ in TXS0828+193.

Actively star-forming galaxies may have strong stellar Balmer absorption lines, in particular for the higher-order Balmer lines. This may result in underestimating the $\mathrm{H} \beta$ emission line flux relative to $\mathrm{H} \alpha$, and thus in overestimating the extinction. Since the continuum in our galaxies is faint we cannot measure the effect of underlying stellar absorption directly. However, the large $\mathrm{H} \alpha$ and $\mathrm{H} \beta$ equivalent widths, the faintness of the continuum, and the significantly larger spatial extent of the line emission indicate that stellar absorption will have a negligible effect.

\subsection{Electron densities}

The [SII] $\lambda \lambda 6716,6731$ doublet in TXS0828+193 is relatively faint and falls into a wavelength range that is dominated by telluric absorption bands; however, in MRC0406-244, the doublet is clearly detected in the $K$ band at signal-to-noise ratios $\approx 10$ (see Fig. 2 for the integrated $K$ band spectrum of MRC0406244). Due to the large line widths, the doublet is partially blended. To minimize the blending, we extracted the integrated spectrum of both bubbles individually, avoiding the central region where lines are particularly broad due to the overlap of the two bubbles. This is justified by the overall very uniform line ratios of the $[\mathrm{SII}] \lambda \lambda 6716,6731$ doublet extracted from smaller apertures.

We show the $K$ band spectra of the two bubbles in Fig. 5. The upper panels show the measured spectra, fits and fit residuals are shown below. The profiles of all lines in the $K$-band are relatively narrow and regular. We used again [OIII] $\lambda 4959,5007$ in the $H$ band to constrain the redshift and line width, so that the line fluxes are the only free parameters.

[SII] line ratios, $R_{[\mathrm{SII}]}=F(6716) / F(6731)$, in the two bubbles are similar within the uncertainties; we measure $R_{[\mathrm{SII}]}^{0406 b}=$ $1.0 \pm 0.15$ in the blueshifted bubble, and $R_{[\mathrm{SII}]}^{0406 r}=1.0 \pm 0.10$ in the redshifted bubble. For a temperature of $T=10^{4} \mathrm{~K}$ (Sect. 4.4), this corresponds to a range in electron density of $n_{\mathrm{e}}^{0406 b}=300-1045 \mathrm{~cm}^{-3}$ and $n_{\mathrm{e}}^{0406 r}=370-830 \mathrm{~cm}^{-3}$ in the blueshifted and redshifted bubble, respectively, with a best value of about $500 \mathrm{~cm}^{-3}$ in either case, which is the value we will adopt in the following.

Only one $z \sim 2 \mathrm{HzRG}$ has an electron density estimate given in the literature, which is MRC1138-262 (Nesvadba et al. 2006b) with densities in the range of $240-570 \mathrm{~cm}^{-3}$. The values in both galaxies are very similar, suggesting that densities of a few $\times 100 \mathrm{~cm}^{-3}$ are likely typical for the luminous optical emission line regions in HzRGs. 

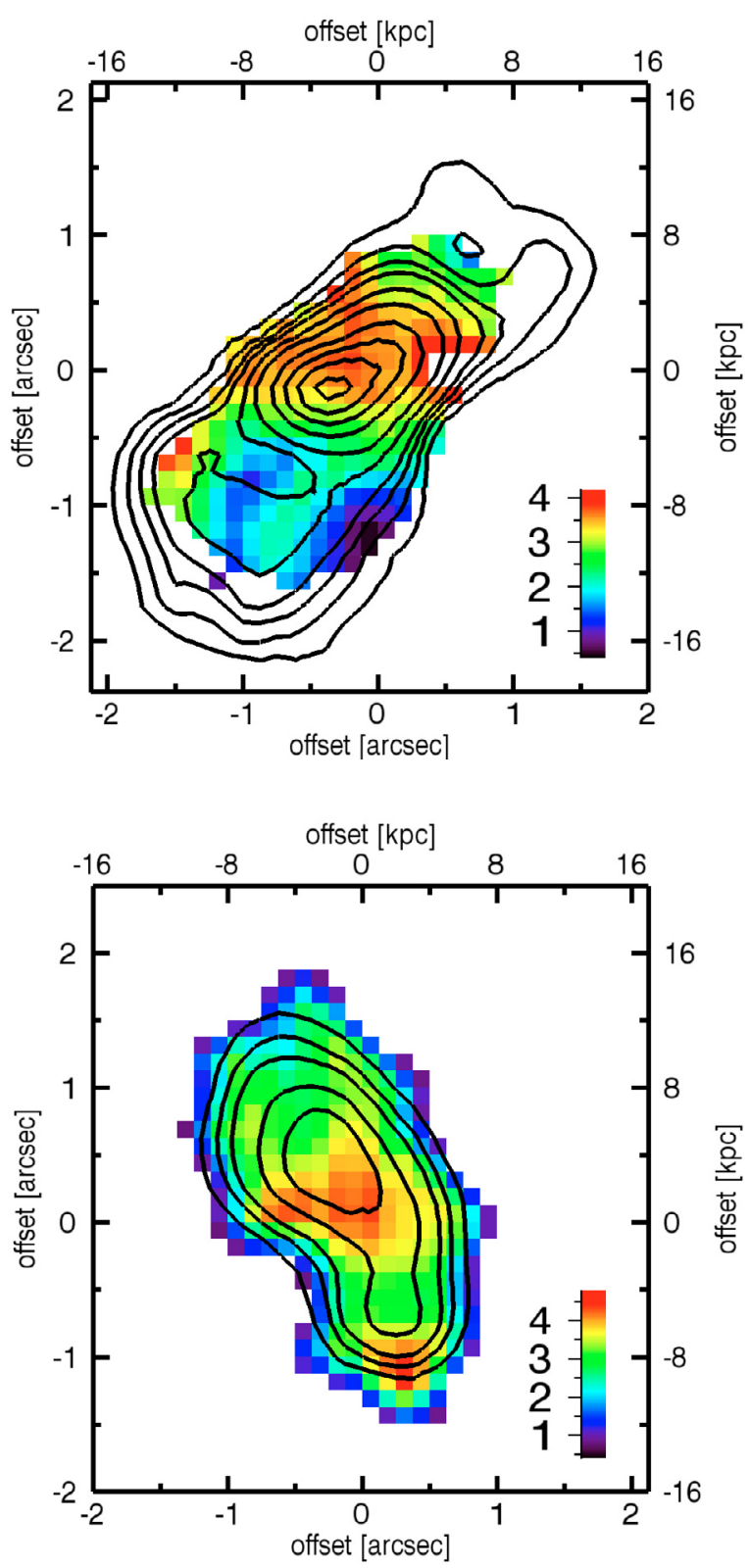

Fig. 4. $A(\mathrm{H} \beta)$ extinction maps for MRC0406-244 (top) and TXS0828+193 (bottom). Color bars show the extinctions (in magnitudes) estimated from the observed $\mathrm{H} \beta / \mathrm{H} \alpha$ emission line ratios, assuming a galactic extinction law. Contours show the emission line morphology to ease orientation.

\subsection{Electron temperatures}

We detect the faint [OIII] $\lambda 4363$ emission line in the $H$-band spectrum of TXS0828+193. We detected the line in a $5 \times 5$ pixel aperture in the $H$ band centered on the bright southern knot. The spectrum around the wavelength of [OIII] $\lambda 4363$ is shown in the inset of Fig. 6. We use the ratio of emission line fluxes $F$ of the 3 [OIII] lines at $4363 \AA$, $4959 \AA$, and $5007 \AA$, respectively, $F_{4363}, F_{4959}$, and $F_{5007}, R_{5007+4959,4363}=\left(F_{4959}+F_{5007}\right) / F_{4363}$ to estimate the electron temperature of TXS0828+193. We find $R_{5007+4959,4363} \sim 173 \pm 52$, correcting for extinction. For an electron density $n_{\mathrm{e}} \sim 500 \mathrm{~cm}^{-3}$, and allowing for $\sim 30 \%$ uncertainty of the $[\mathrm{OIII}] \lambda 4363$ flux measurement, this corresponds to an electron temperature of $\sim 10100-12500 \mathrm{~K}$ (see Fig. 6).

We do not detect [OIII] $\lambda 4363$ or $\mathrm{H} \gamma$ in MRC0406-244. This may be due to the somewhat lower redshift of this source,

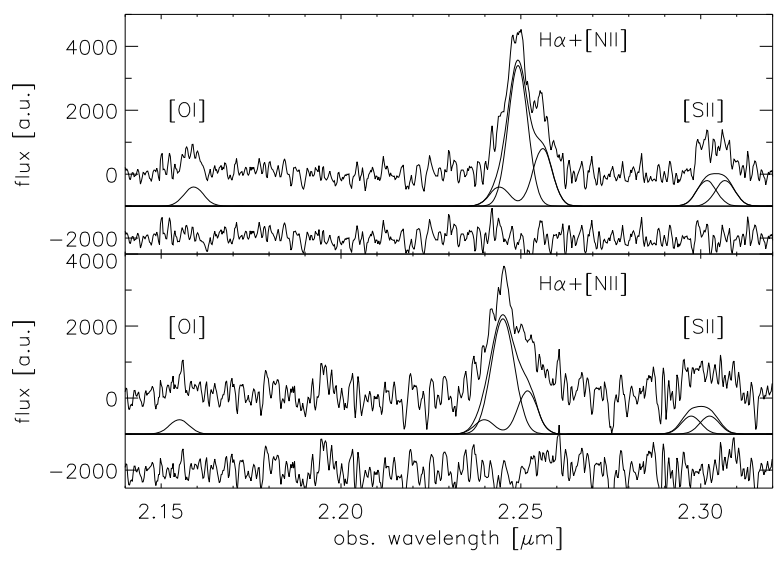

Fig. 5. Integrated spectra of the two bubbles in MRC0406-244, the redshifted southern bubble is shown on top, the blueshifted northern bubble is shown below. Upper panels show the spectra, Gaussian fits to the emission lines and fit residuals are shown in the mid and lower panels, respectively. Spectra are smoothed by 5 pixels spectrally, corresponding to $\sim 1 / 5$ of the total line width. The measured [SII] $\lambda \lambda 6716,6731$ line ratios are consistent with electron densities of a few $100 \mathrm{~cm}^{-3}$, with a best-fit value of $500 \mathrm{~cm}^{-3}$ (see text). Redshifts and line widths were measured from the [OIII] $\lambda \lambda 4959,5007$ line doublet, so that the only free parameters to all other lines, including [SII], are the line fluxes.

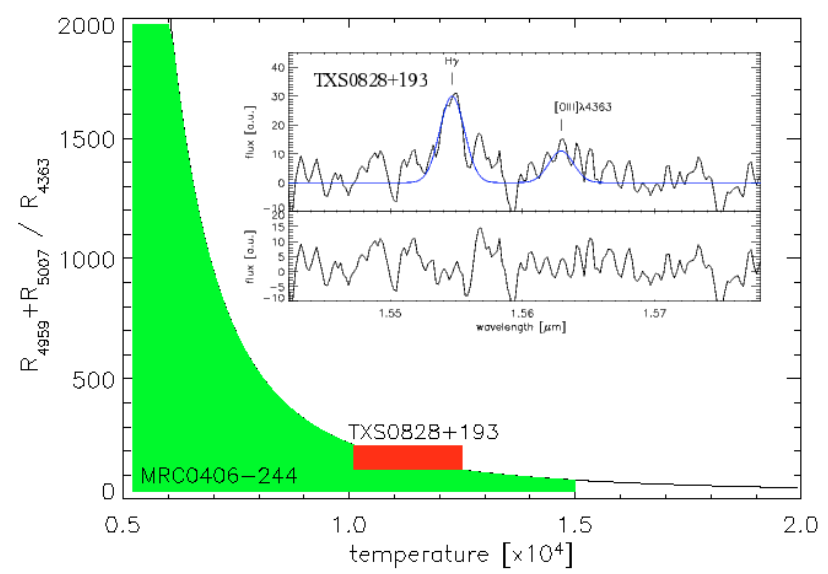

Fig. 6. Relationship between electron temperature and [OIII] line ratios for an electron density of $500 \mathrm{~cm}^{-3}$. The green shaded area shows the temperature range for MRC0406-244 derived from a $3 \sigma$ limit on $[\mathrm{OIII}] \lambda 4363$, the red area shows the range of temperatures from the ratio based on the measured [OIII] $\lambda 4363$ flux in TXS0828+193, allowing for the $30 \%$ uncertainty. The inset shows a zoom on $\mathrm{H} \gamma$ and [OIII] $\lambda 4363$ in the $H$-band spectrum of TXS0828+193. Blue lines indicate the expected emission lines with the redshift and line width measured from [OIII] 25007 . The lower panel shows the fit rediduals.

which causes both lines to fall at a wavelength where the transparency of the night sky and SINFONI's efficiency are not very favourable. Placing a $3 \sigma$ limit on [OIII] $\lambda 4363$ in MRC0406-244, we find an extinction-corrected $R_{5007+4959,4363}=55.6$, which implies an electron temperature $T_{\mathrm{e}}^{0406} \leq 1.5 \times 10^{4} \mathrm{~K}$.

Measuring electron temperatures from $R_{[\mathrm{OIII}]}$ is the most direct method, but obviously requires high-quality spectroscopy of high-redshift galaxies, and exposure times that are often prohibitively large. Humphrey et al. (2008) explored alternative, temperature-dependent line ratios using longslit spectra of rest-frame UV and optical emission lines. For MRC0406244 and TXS0828+193, which are both included in their sample, they find $T_{\mathrm{e}}^{0406}=11700_{-1500}^{+1800} \mathrm{~K}$ and for several line 
ratios in TXS828+193, they find temperatures in the range $T_{\mathrm{e}}^{0828}=14000-22000 \mathrm{~K}$. While their results differ from ours if taken at face value, the differences are insignificant, given the large uncertainties of either measurement.

\subsection{Ionized gas masses}

We use the extinction corrected $\mathrm{H} \alpha$ fluxes (Sect. 4.2) to estimate ionized gas masses. Similar to Nesvadba et al. (2006b), we assume simple case B recombination, and use the $n_{\mathrm{e}} \sim$ $500 \mathrm{~cm}^{-3}$ electron density measured in MRC0406-244 (see also Sect. 4.2 of Nesvadba et al. 2006b, in particular Eq. (1)). Total $\mathrm{H} \alpha$ fluxes in MRC0406-244 and TXS0828+193 correspond to $\log \left[L / L_{\odot}\right]_{\text {corr }}^{0406}=45.2 \mathrm{erg} \mathrm{s}^{-1}$ and $\log \left[L / L_{\odot}\right]_{\text {corr }}^{0828}=$ $45.3 \mathrm{erg} \mathrm{s}^{-1}$, respectively (or $\log \left[L / L_{\odot}\right]^{0406}=44.5 \mathrm{erg} \mathrm{s}^{-1}$ and $\log \left[L / L_{\odot}\right]^{0828}=44.4 \mathrm{erg} \mathrm{s}^{-1}$ without extinction correction). Estimating the gas masses in each spatial resolution element, and summing over the full emission of the two galaxies, we find $\log \left[M_{\mathrm{HII}} / M_{\odot}\right]^{0406} \sim 10.6$ and $\log \left[M_{\mathrm{HII}} / M_{\odot}\right]^{0828} \sim 10.7$ for MRC0406-244 and TXS0828+193, respectively. All values are also given in Table 7.

We caution that ionized gas mass estimates at $z \geq 2$ can be precise to an order-of-magnitude level only. In particular, extinction and electron densities involve measuring relatively faint lines, $\mathrm{H} \beta$ and $[\mathrm{SII}] \lambda \lambda 6716,6731$, and the physical parameters are strongly non-linear functions of the line ratios. In spite of signal-to-noise ratios $\sim 10$ in the faint lines, which is excellent compared to IFU studies of galaxies at high redshift (e.g. Förster Schreiber et al. 2006) this will add uncertainties of factors $\geq 2$ in each estimate and for typical values measured in our sample. Correcting for the blend of the $\mathrm{H} \alpha+[\mathrm{NII}]$ lines, we minimized the contribution of $\mathrm{H} \alpha$ in the fit, which will result in underestimating the $\mathrm{H} \alpha$ flux. Overall, we find that these uncertainties will amount in total to less than \pm 0.5 dex, safely placing our estimates into the range of a few $\times 10^{10} M_{\odot}$.

A few studies have previously estimated ionized gas masses in HzRGs based on slit-spectra and narrow line images, although with significantly larger uncertainties. Armus et al. (1998) report on a giant nebulosity in $4 \mathrm{C}+19.71$ at $z \sim 3.6$, which they found through [OIII] $\lambda 5007$ narrow band imaging, estimating gas masses of $\sim 10^{8-9} M_{\odot}$. From [OII] $\lambda 3727$ longslit spectroscopy of a sample of $52 \mathrm{HzRG}$ s taken from the 3CR, Baum \& McCarthy (2000) find that the ionized gas masses in HzRGs increase linearly with redshift, which may be related to larger quantities of cold gas in and around massive galaxies at high redshift. They also find that the $10 \mathrm{HzRG}$ at $z \geq 1$ have ionized gas masses of several $10^{9} M_{\odot}$, which is likely a lower limit, due to partial coverage of the source with the slit, and because [OII] $\lambda 3727$ is susceptible to extinction. (In Sect. 4.2 we found $A_{V}=1-4 \mathrm{mag}$ for the extended emission line regions of our targets.) Comparing their result for MRC0406-244 and ours, we can loosely estimate that correction factors of a few are required to compensate for systematic offsets. This suggests that the intrinsic masses of their sample will plausibly be of order $10^{10} M_{\odot}$.

Humphrey et al. (2008) measured $\mathrm{H} \alpha$ and $\mathrm{H} \beta$ fluxes for 9 powerful radio galaxies during the "Quasar Era" with the ISAAC longslit spectrograph on the VLT. Unfortunately, $\mathrm{H} \beta$ is not or only marginally detected in most of their sample, making a direct comparison with the extinction-corrected gas masses of our sample difficult. However, for the uncorrected $\mathrm{H} \alpha$ fluxes, we find similar values within factors of a few as in our sample, which suggests ionized gas masses of order $10^{10} M_{\odot}$ may be common in HzRGs.

\section{The nature of the emission line region}

Rest-frame optical integral-field spectroscopy has been published for a few dozen galaxies at $z \sim 2$ of different types and masses (e.g. Förster Schreiber et al. 2006; Nesvadba et al. 2007b, 2006a; Swinbank et al. 2006; Law et al. 2007; Nesvadba et al. 2008; Genzel et al. 2006; Wright et al. 2007; Bournaud et al. 2008), including galaxies undergoing mergers, candidates for relatively quiescent disk rotation and galaxies that are likely disks in formation, and galaxies showing the signs of starburstdriven winds. The sample includes galaxies with and without AGN. Compared to these samples, our radio galaxies have several unique properties which allow us to constrain the mechanism which is driving the gas dynamics. We will briefly discuss the different possible scenarios.

\subsection{Merging}

If the two bubbles represented the interstellar medium of two massive galaxies in course of a merger, we should find a continuum peak in each bubble, representing the stellar component of each galaxy of the merging pair. However, we find only one continuum source in MRC0406-244 and TXS0828+193, respectively, which is in the center of each source, where the velocity of the ionized gas changes abruptly between the blueshifted and the redshifted bubble. (For MRC0316-257, we did not detect the continuum.) This does not rule out that these galaxies are related to a major merger, however, the two components must be closer than the resolution of our data, about $5 \mathrm{kpc}$, and the two bubbles cannot be gravitationally bound to one of the galaxies each. (Note that our continuum images are not deep enough to rule out minor mergrs.)

Broad-band morphologies of HzRGs are in agreement with this interpretation. Broad-band morphologies of HzRGs are known to be often irregular (e.g., van Breugel et al. 1998; Pentericci et al. 1999), however, in many cases and depending on redshift, this may be due to emission line contamination, as has been suspected before (e.g. Rush et al. 1997), and is now evident from the line and continuum morphologies extracted from our data cubes. A number of HzRGs, including galaxies with HST-NICMOS imaging, have very compact morphologies and elliptical light profiles consistent with being merger remnants (van Breugel et al. 1998; Pentericci et al. 1999).

\subsection{Disk rotation}

Disk rotation and merging are difficult to distinguish at $z \sim 2$ due to the relatively coarse spatial resolution of the data relative to the relevant size scales, typically several seconds of arc (see Nesvadba et al. 2008, for a more detailed discussion). However, all candidates for disk rotation at high redshift have more gradual velocity offsets. Unlike other high-redshift galaxy populations, the emission line regions of HzRGs are well resolved spatially, and should show a characteristic velocity pattern known as "spider diagram" if their kinematics were dominated by disk rotation (see, e.g. Fig. 1 of Kronberger et al. 2007). However, the observed abrupt velocity offset within a spatially unresolved region characterized by strong line splitting (Fig. 7) is at odds with galaxies showing disk rotation at low and at high redshift. In addition, the emission line morphologies are often irregular or filamentary, which would not be typical for rotating gaseous disks (see Figs. 8 to 10).

Moreover, the large velocity offsets and line widths suggest unplausibly large enclosed masses of often $10^{13-14} M_{\odot}$ within 


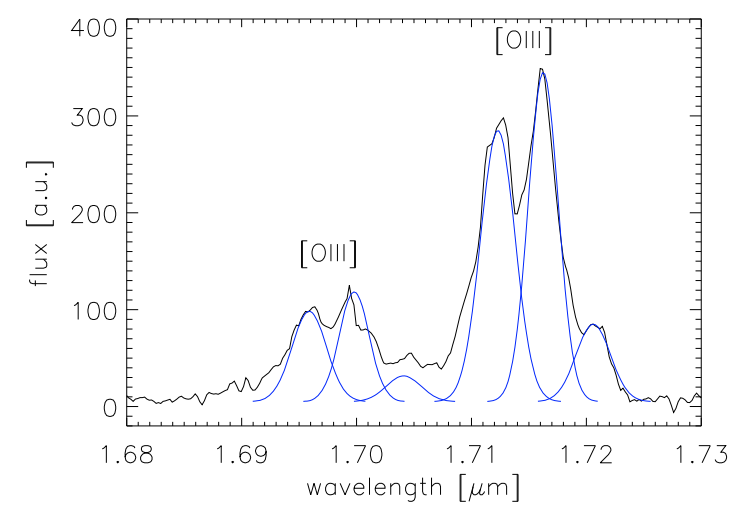

Fig. 7. [OIII] emission lines extracted from a small aperture $\left(0.375^{\prime \prime} \times\right.$ $\left.0.375^{\prime \prime}\right)$ near the center of MRC0406-244. Line splitting is evident in both lines, with three narrow components and an underlying, broad component. The mid component in [OIII] $\lambda 4959$ is affected by a night sky line residual. To guide the eye, we also show Gaussians for each component of the [OIII] $\lambda 4959$ and [OII] $\lambda 5007$ line respectively. Note that these are not strictly fits, but have the same line width and redshift for both [OIII] lines. The red component of this line appears fainter than the corresponding component of the $[\mathrm{OIII}] \lambda 5007$ line, because of an underlying broad component of the [OIII] $\lambda 5007$ line superimposed, which we ignored.

few $10 \mathrm{~s}$ of kpc (compared to stellar masses of few $\times 10^{11} M_{\odot}$; Seymour et al. 2007), if the gas was to be bound gravitationally (see, e.g., Table 1 of Baum \& McCarthy 2000). For HzRGs that are surrounded by galaxy overdensities, Venemans et al. (2007) estimate virial masses of few $\times 10^{14} M_{\odot}$ for the entire dark matter structure out to radii of $\sim 1-2 \mathrm{Mpc}$, based on observations of the velocity dispersion of the companion galaxies. If the observed emission line kinematics of HzRGs were due to rotation, then a significant fraction of this mass would have to be within the inner 20-30 kpc of the structure, at odds with what we know about dark matter halo mass profiles. Nesvadba et al. (2006b) argued that the ionized gas of MRC1138-262 has a kinetic energy which is about similar to the binding energy of a galaxy with a mass of few $\times 10^{11} M_{\odot}$, in which case much of the gas would not be gravitationally bound. We conclude that the observed velocity fields cannot be related to gravitationally driven motion, they are super-gravitational.

\subsection{Outflows or infall?}

Directly measuring the direction of a gas flow in extragalactic objects is inherently difficult, in particular at high redshift. Observed velocity gradients are near the escape velocity of the dark halo surrounding a massive galaxy (see Nesvadba et al. $2006 \mathrm{~b}$, for a more detailed discussion), which suggests that we may be witnessing the ionized gas being unbound from the host galaxy. In the next section we will argue that the gas dynamics are most likely related to the expanding cocoon of hot, overpressurized gas surrounding the radio jet in agreement with previous studies based on longslit spectroscopy (e.g., Clark et al. 1998; Villar-Martín et al. 1999; Inskip et al. 2002; Best et al. 1997).

Such an interaction will result in complex kinematics, in agreement with the observed large line widths. The cocoon likely forms from backflowing gas which has previously been heated by shocks at the working surface of the jet. Although Alexander \& Pooley (1996) pointed out that this gas will likely not move far from the hotspot and may mostly contribute to inflate the cocoon, this makes a more detailed assessment of the flow direction of the ionized gas worthwhile.

One way to constrain this direction is through a careful study of the radio spectral properties of the two lobes in each source (see also Humphrey et al. 2008). Radio emission from a source embedded in a magnetized plasma will be depolarized by a degree that depends on the column of the transversed medium. Thus, the near side of the radio source will appear more strongly polarized than the far side (the "Laing-Garrington effect"; Laing 1988; Garrington et al. 1988). Moreover, the observed brightness of the radio hotspots on the near side of the radio source may be enhanced due to Doppler-boosting (e.g., Alexander \& Leahy 1987). The hotspots have younger ages and accordingly flatter spectral indices than the surrounding lobes. Thus, at high frequencies, the relative contribution of the hotspot to the overall emission from the lobe will be larger, and the spectral index on the near side of the radio source will appear flatter than that on the far side.

Comparing with the observations of Carilli et al. (1997), we find in each of our galaxies that the northern radio lobe is more strongly polarized at $4.7 \mathrm{GHz}$ and at $8.2 \mathrm{GHz}$ than the southern lobe. Moreover, the northern lobe has a somewhat flatter spectral index in all galaxies. In all three cases, this is also the side of the blueshifted bubble of ionized gas. This strongly advocates that the blueshift represents a net outflow relative to the central galaxy.

Outflows of gaseous material are also observed in powerful radio galaxies at lower redshifts, $z<0.1$. Morganti et al. (2005) find broad, blueshifted HI absorption lines at relative velocities of $\geq 1000 \mathrm{~km} \mathrm{~s}^{-1}$ from the systemic redshift in 7 out of 8 sources. This is consistent with AGN-driven outflows of neutral gas, corresponding to mass loss rates of up to $\sim 50 M_{\odot} \mathrm{yr}^{-1}$.

With the simple premise that the extended emission line regions in HzRGs represent energetic outflows, we will now constrain the basic dynamics of the gas, before arguing in the next section, that the outflows are most likely related to the radio jet.

\subsection{Dynamical time scales}

Emission line regions extend over fairly similar radii in all three galaxies, $10 \mathrm{kpc} \times 9 \mathrm{kpc}, 15 \mathrm{kpc} \times 9 \mathrm{kpc}$, and $14 \mathrm{kpc} \times 9 \mathrm{kpc}$ for MRC0316-257, MRC0406-244, and TXS0828+193, respectively (see Table 6). We can use the size of the outflows along the axis of the radio jet and the relative velocity between the blueshifted and the redshifted bubble in each galaxy to estimate the crossing time. Using the velocities given in Table 6 , we find dynamical timescales of $\tau_{\text {ion }} \sim$ few $\times 10^{7} \mathrm{yrs}$, which is similar to ages inferred for radio jets (e.g., Kaiser \& Alexander 1997). See Table 6 for the ages derived for individual galaxies. These estimates can only be accurate within factors of a few, given the unknown radial density profile of the gas prior to the radio-loud phase, the effects of projection and relatively coarse spatial resolution of our data.

Alternatively, we can assume that the velocity gradients observed in individual bubbles are dominated by the lateral expansion of the bubbles, as observed in low-redshift narrow-line regions being inflated by interactions with the overpressurized, expanding cocoon of the radio jet (e.g., Capetti et al. 1999). HzRGs have very irregular environments with gaseous halos (e.g., Villar-Martín et al. 2003), satellite galaxy overdensities (e.g. Venemans et al. 2007), and perhaps infalling gas clouds, which will influence the distribution and density of the sourrounding medium. This will make it difficult to reliably measure the relatively small velocity gradients within an individual 

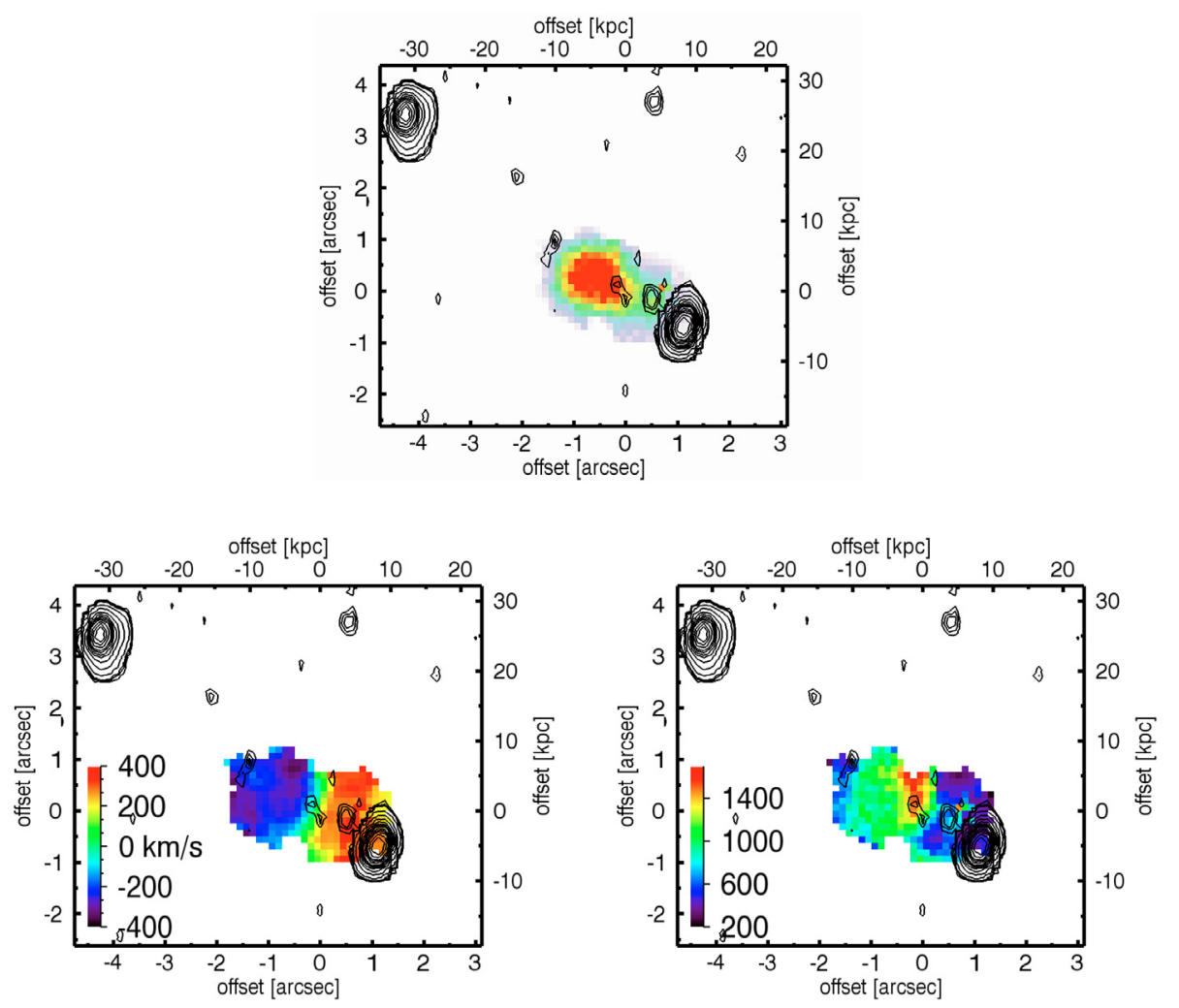

Fig. 8. Top: $[\mathrm{OIII}] \lambda 5007$ emission line morphology of MRC0316-257 at $z=3.13$ with contours showing the $1.4 \mathrm{GHz}$ morphology. Bottom left: velocity map. Color bar shows the relative velocities in $\mathrm{km} \mathrm{s}^{-1}$, contours indicate the $1.4 \mathrm{GHz}$ morphology. Bottom right: maps of the line widths, color bars show the FWHM in $\mathrm{km} \mathrm{s}^{-1}$, contours indicate the $1.4 \mathrm{GHz}$ morphologies. North is up, east to the left in all images.
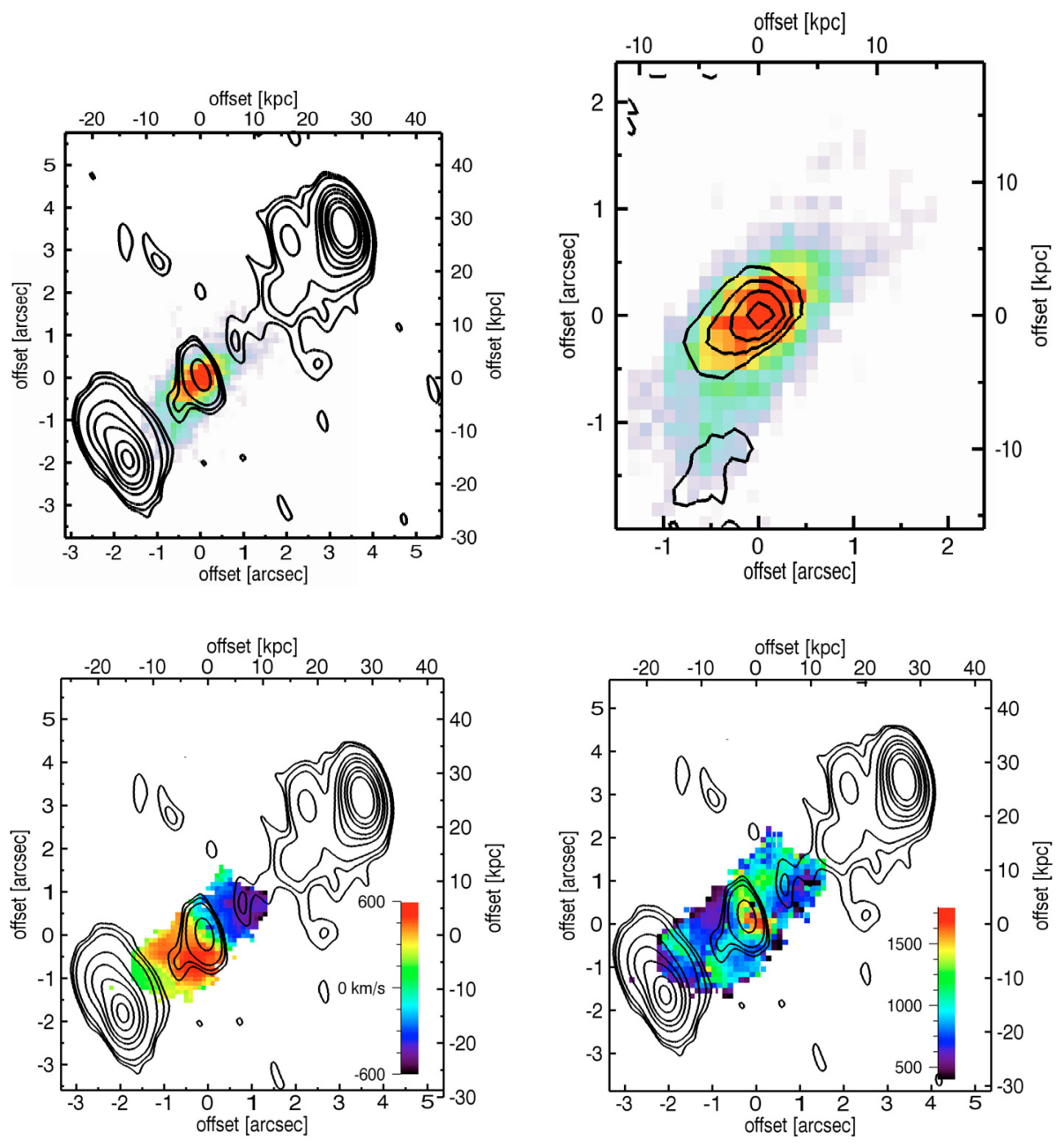

Fig. 9. Top: $[\mathrm{OIII}] \lambda 5007$ emission line morphology of MRC0406-244 at $z=2.42$ with contours showing the $1.4 \mathrm{GHz}$ morphology. Top right: [OIII] $\lambda 5007$ emission line morphology with contours showing the line-free restframe optical continuum. Bottom left: velocity map. Color bar shows the relative velocities in $\mathrm{km} \mathrm{s}^{-1}$, contours indicate the $1.4 \mathrm{GHz}$ morphology. Bottom right: maps of the line widths, color bars show the $F W H M$ in $\mathrm{km} \mathrm{s}^{-1}$, contours indicate the $1.4 \mathrm{GHz}$ morphologies. North is up, east to the left in all images. 

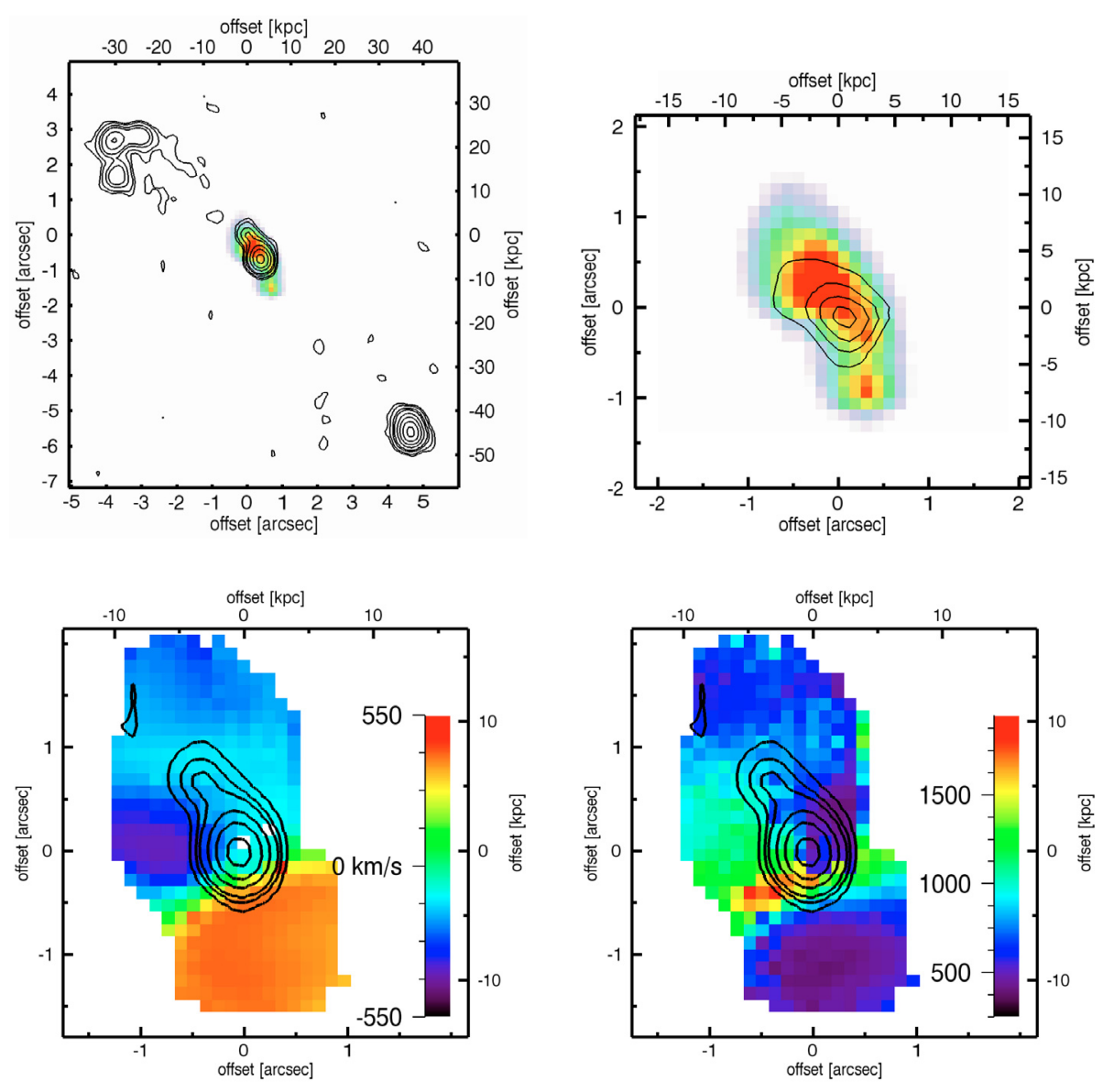

Fig. 10. Top: $[\mathrm{OIII}] \lambda 5007$ emission line morphology of TXS0828+193 at $z=2.57$ with contours showing the $1.4 \mathrm{GHz}$ morphology. Top right: [OIII] $\lambda 5007$ emission line morphology with contours showing the line-free rest-frame optical continuum. Bottom left: velocity map of TXS0828+193. Color bar shows the relative velocities in $\mathrm{km} \mathrm{s}^{-1}$, contours indicate the $1.4 \mathrm{GHz}$ morphology. Bottom right: maps of the line widths, color bars show the FWHM in $\mathrm{km} \mathrm{s}^{-1}$, contours indicate the $1.4 \mathrm{GHz}$ morphologies. North is up, east to the left in all images.

bubble. However, if we only use clouds with relatively smooth internal velocity gradients, in particular the blue (northern) bubbles of MRC0316-247 or of MRC0406-244, we find internal velocity gradients of $\sim 120-300 \mathrm{~km} \mathrm{~s}^{-1}$, corresponding to dynamical time scales of a few $\times 10^{7} \mathrm{yrs}$, which is consistent with the dynamical time scales estimated from the velocity gradients along the radio jet axis.

\subsection{Kinetic energies}

In Sects. 5.2 and 5.3 of Nesvadba et al. (2006b), we applied two methods to estimate the injection of kinetic energy into the outflows:

(1) A strict, but presumably not very precise lower limit, based on the observed ionized gas mass, the bulk motion, $v$, and the turbulent motion, $\sigma$, i.e., $E=1 / 2 m\left(v^{2}+\sigma^{2}\right)$.

Velocities and line widths are taken from the kinematic maps shown in Figs. 8 to 10 and thus represent the local values for each spatial resolution element. We discarded the very broad lines near the center of the galaxy, which we believe are largely due to overlaps between the red and the blue bubble. Within each bubble, line widths are larger than the velocity changes, so that they give a fair representation of the intrinsic line widths within the spatial resolution of our data. Since we neglected the likely presence of other gas phases, as well as uncertainties related to projection, this can only be a conservative lower limit. We do not assume pressure equilibrium, but that velocities are dominated by ram pressure.

(2) An energy estimate based on the assumption that the ionized gas is being entrained and accelerated through adiabatic inflation of a bubble of hot overpressurized material, which follows, e.g., Dyson \& Williams (1980), and which yields $\dot{E}=1.5 \times 10^{46} r_{10}^{2} v_{1000}^{3} n_{0.5} \mathrm{erg} \mathrm{s}^{-1} \cdot r_{10}$ indicates the size of the emission line nebulae in units of $10 \mathrm{kpc}, v_{1000}$ the velocity in units of $1000 \mathrm{~km} \mathrm{~s}^{-1}$, and $n_{0.5}$ the ambient gas density of the warm interstellar medium prior to being swept up by the bubble in multiples of $0.5 \mathrm{~cm}^{-3}$. This approach is highly idealized given the complexity of the turbulence and bulk motion observed and expected from hydrodynamical simulations of radio jets (e.g., Krause 2005; Saxton et al. 2005; Gaibler et al. 2007; Sutherland \& Bicknell 2007), but may serve as a rough analytical surrogate to more detailed estimates. We used the velocity maps shown in Figs. 8 to 10 to estimate the total velocity offsets between bubbles, and set the expansion velocity in each bubble to half the total value. Values for individual galaxies are given in Table 6.

Although our estimates of velocities and line widths are precise to $10-20 \%$, each approach is based on a set of very simple assumptions. While this is appropriate given the observational difficulties of high-redshift studies, it does introduce astrophysical uncertainties that will make each estimate precise to factors of a few only.

Applying method (1) to our present data set, we find energy injection rates of $3 \times 10^{43} \mathrm{erg} \mathrm{s}^{-1}$ and $1.3 \times 10^{44} \mathrm{erg} \mathrm{s}^{-1}$ for TXS0828+193 and MRC0406-244, respectively, for mass estimates without extinction correction. This represents a very conservative lower limit on the energy injection rate. If using extinction-corrected mass estimates instead, we find energy injection rates of $\sim 2 \times 10^{45} \mathrm{erg} \mathrm{s}^{-1}$ are required for both galaxies with ionized mass estimates, MRC0406-244 and TXS0828+193. (Individual values are given in Table 6.) 
For MRC0316-257, we do not have an explicit estimate of the ionized gas mass, because at the redshift of the source, $z \sim 3.1, \mathrm{H} \alpha$ cannot be observed from the ground, and $\mathrm{H} \beta$ is very faint. The velocity maps shown in Figs. 8 to 10 , however, suggest overall very similar properties. Under the premise, that the ionized gas mass will not greatly differ, we would hence expect to also find similar estimates of the kinetic energy injection rate. For the 10 galaxies in the sample of Baum \& McCarthy (2000) at $z \geq 1$, we find very similar results. Using their measurements of the ionized gas masses, velocities, and line widths and our method 1, we find total energy injections of $10^{57-59}$ erg over dynamical timescales of about $10^{7}$ yrs. Note that this is a strict lower limit. If we correct for extinction as found from our SINFONI data set, and uncertainties in the alignment between the longslit and the direction of largest velocity shear, these estimates will easily increase by about an order of magnitude. This certainly highlights the ubiquity of powerful outflows of ionized gas in HzRGs.

Method (2) does not depend on the ionized gas mass, and can therefore be applied to all three galaxies. We used the velocity maps shown in Fig. 8 to estimate the total velocity offsets between bubbles, and set the expansion velocity in each bubble to half the total value. Values for individual galaxies are given in Table 6.

We find that energy injection rates of $\dot{E} \sim 0.7-4 \times$ $10^{45} \mathrm{erg} \mathrm{s}^{-1}$ are required to power the outflows (see Table 6 for individual values). Note that velocities are not corrected for orientation effects. Assuming that inclinations will typically be around $45^{\circ}$ or higher, intrinsic velocities may be factors 2-3 higher, which would result in kinetic energies of up to several $\times 10^{46} \mathrm{erg} \mathrm{s}^{-1}$ and total kinetic energy injections during an AGN lifetime of order $10^{60} \mathrm{erg}$.

\section{The role of the radio jet}

\subsection{Kinematics}

Our study provides direct evidence for outflows of ionized gas driven by powerful AGN during the "Quasar Era". Nesvadba et al. (2006b) argued in the case of MRC1138-262 that the energy injection, mass outflow rates, and dynamical time scales satisfy the criteria set by observations of massive galaxies at low redshift. In particular, the outflow time scales are short enough to explain the observed relative enhancement of $\alpha$ elements over iron as a function of velocity dispersion in early-type galaxies (e.g., Pipino \& Matteucci 2004; Pipino et al. 2007). Direct studies of galaxies undergoing phases of strong AGN feedback like HzRGs are ideal to investigate by which mechanism the energy output of the AGN is being converted into thermal and kinetic energy of the surrounding cold gas. Broadly speaking, two groups of processes have been proposed in the literature (1) outflows driven by the radiative energy output of the AGN (e.g., King 2003; Sazonov et al. 2005; Murray et al. 2005; Ciotti \& Ostriker 2007); (2) outflows related to mechanical interactions with the relativistic, synchrotron-emitting plasma of radio-loud AGN. These were mainly proposed to overcome the cooling-flow problem (e.g., Binney \& Tabor 1995; Ruszkowski \& Begelman 2002; Kaiser \& Binney 2003), but are also addressing the impact of the jets in individual radio galaxies (e.g., Mellema et al. 2002; Fragile et al. 2004; Saxton et al. 2005; Krause 2005; Sutherland \& Bicknell 2007; Krause \& Alexander 2007).

While both classes rely on physically plausible mechanisms, their actual impact on the surrounding gas depends strongly on the efficiency by which the energy output of the AGN is being converted into thermal and kinetic energy of the surrounding gas. This is best addressed through direct observations of systems that are in a phase dominated by powerful, AGN driven outflows, like the galaxies of our sample. Overall our analysis favors a picture where the radio jet plays a dominant role in driving the gas, based on arguments related to the observed geometry, timescales, and kinetic energy of the flows.

Orientation relative to the jet axis: in the 3 galaxies of our sample, the extended emission line regions are elongated along the axis of the radio jets, and are well aligned with the jet axis. The same is suggested by the overall velocity field of the galaxies - we clearly distinguish a redshifted and a blueshifted bubble, suggesting a back-to-back flow, with a sharp transition near the center of the galaxy. The size of the transition regions appears dominated by the spatial resolution of our seeing-limited data. In the previous section, we also showed that the blueshifted gas appears to be aligned with the near side of the radio source in all three cases. This is a prerequisite, if the ionized gas is being accelerated away from the galaxy by the expanding cocoon of hot gas surrounding the radio jet.

Spatial extent: the region of high surface brightness emission extends to radii that are smaller than the size of the radio lobes. Extending this argument, Nesvadba et al. (2007a) find that galaxies with compact radio sources do not have spatially extended line emission, but kinetic properties suggesting that the gas kinematics are driven by the same underlying processes. In agreement with spectral studies of radio jets (e.g. Murgia et al. 1999; Owsianik \& Conway 1998), this is a natural result if compact radio sources are predominantly young. For similar entrainment rates as found in our sample, and typical ages of a few $\times 10^{5-6}$ yrs estimated for compact radio sources, these galaxies should not yet have entrained substantial fractions of the interstellar medium, in agreement with observations (see Nesvadba et al. 2007a, for details).

Villar-Martín et al. (2002, 2003, 2006, 2007) find faint restframe UV emission surrounding HzRGs, with line widths and velocity gradients that suggest that the gas does not participate in the violent processes in the inner halo, that we are tracing with our rest-frame optical data. These kinematically quiescent outer halos are always found at radii beyond the radio jets, which is highly suggestive in a scenario where the outflows are dominated by interactions with the radio jet.

Time scales: in Sect. 5.4, we estimated dynamical time scales of a few $\times 10^{7}$ yrs from the characteristic velocities and spatial extent of the flows. In spite of large uncertainties, this is in the typical range of ages estimated for radio jets (e.g., Kaiser \& Alexander 1997; Kaiser \& Best 2007; Blundell \& Rawlings 1999).

Energies: anticipating the results of Sect. 7.4, we estimate that about $10 \%$ of the kinetic energy flux of the jet is transformed into kinetic energy of the gas. This is fully consistent with a picture where the radio jet is the primary driver of the observed gas kinematics.

These large coupling efficiencies may be somewhat surprising. Early models of radio jets indicated that powerful jets like the ones in our sample (in the energy range of FR II radio galaxies), will pass through the ambient medium rather undisturbed, while low-power jets (in particular FR I galaxies) may deposit most of their energy in the surrounding medium after being disrupted (e.g., De Young 1991).

More recent models with better resolution, and larger density contrast between jet plasma, ambient medium, and embedded clouds, suggest a more subtle picture. Large jet kinetic energies 
of few $\times 10^{46} \mathrm{erg} \mathrm{s}^{-1}$ and highly inhomogeneous ambient media are clearly required to adequately describe powerful radio galaxies at $z \sim 2$, and several recent models have now investigated this (e.g. Saxton et al. 2005; Sutherland \& Bicknell 2007; Krause 2005; Krause \& Alexander 2007). The basic properties of the simulated cocoons are not unrealistic compared to what is observed, e.g., internal velocities of order $1000 \mathrm{~km} \mathrm{~s}^{-1}$ or aspect ratios of $\sim 3$ between the radial and the lateral axis of the cocoon (Saxton et al. 2005; Krause 2005).

However, the model parameters are adapted to lower redshift galaxies with somewhat lower jet eneriges, and lower fractions of cold gas. In particular, the environments of HzRGs suggest the galaxies are surrounded by halos of cold gas with complex substructure, rather than well-settled disks of cold gas as adopted by Sutherland \& Bicknell (2007).

Saxton et al. (2005) point out that a clumpy environment may destabilize or even disrupt the jet by scattering on several clouds, and through Kelvin-Helmholtz instabilities induced by the overall level of turbulence rather than individual jetcloud interactions. It is plausible that this will enhance the efficiency with which the kinetic jet energy is being deposited into the cold gas even for the most powerful jets. Models better adapted to the properties of powerful radio galaxies at $z \sim 2$ may be an interesting way of investigating this further, including the kinematic constraints now available from integral-field spectroscopy.

\section{Impact on the multiphase gas content of HzRGs}

\subsection{Mass budgets}

The extended emission line regions of HzRGs do not only have extreme kinematic properties, but also an unusually large content of ionized gas, few $\times 10^{10} M_{\odot}$ (Sect. 4.5 , see also Baum \& McCarthy 2000). This exceeds the amount of ionized gas in any other high-redshift galaxy population discussed in the literature by several orders of magnitude.

For example, the same mass estimate applied to the total $\mathrm{H} \alpha$ emission of $z \sim 2$ actively star-forming "BX"-galaxies measured by Erb et al. (2006), would only correspond to a few $\times 10^{7} M_{\odot}$, and most of this gas is probably related to star-forming regions and does not participate in an outflow. Nesvadba et al. (2007b) used SINFONI to obtain spatially-resolved data of the starburst-driven wind in a submillimeter selected, strongly starforming galaxy (SMG), SMMJ14011+0252. They find that in spite of high star-formation rates of $S F R \sim 300 M_{\odot} \mathrm{yr}^{-1}$, only $\sim 10 \%$ of the total $\mathrm{H} \alpha$ flux is in a blueshifted component - the wind - which would correspond to ionized gas masses of a few $\times 10^{6} M_{\odot}$.

Overall, the stellar masses and star-formation rates in $z \sim 2$ HzRGs closely resemble those of SMGs at similar redshifts. Moreover, several $10^{10} M_{\odot}$ of cold molecular gas traced by CO millimeter line emission have been observed in SMGs (e.g., Neri et al. 2003; Greve et al. 2005) as well as in HzRGs (e.g., Papadopoulos et al. 2000; De Breuck et al. 2003, 2005; Klamer et al. 2005). Large masses of cold gas are a necessary prerequisite to fuel a strong starburst. However, not all HzRGs have been detected in CO. For TX0828+193 specifically, which appears to have several $10^{10} M_{\odot}$ in ionized gas (Sect. 4.5), Nesvadba et al. (in prep.) did not detect CO line emission, but place an upper limit of $\sim 1 \times 10^{10} M_{\odot}$ in cold molecular gas within the galaxy (for a ULIRG-like conversion factor from CO luminosity to $\mathrm{H}_{2}$ mass, similar to that adopted for other high-redshift
CO-luminous galaxies). Taken at face value, this is less than the amount of ionized gas we observe with SINFONI. Thus, it appears that the ratios of ionized to molecular gas in HzRGs, are of order unity or greater, $R_{\text {ion,mol }}^{\mathrm{HzRGs}} \geq O(1)$, compared to $\left.R_{\text {ion,mol }}^{\mathrm{SFG}} \leq\right)\left(10^{-(3-4)}\right)$ for actively star-forming galaxies at $z \sim 2$ (SFGs) including SMGs. (Either value is affected by observational uncertainties of factors of a few, but the large contrast between star-forming galaxies and radio galaxies makes this comparison robust nonetheless.) This certainly highlights the importance of the observed outflows for the evolution of the host galaxy, and it is interesting in this context that some HzRGs show the signs of strong star formation (like large reservoirs of molecular gas, strong PAH and submillimeter emission, Archibald et al. 2001; Reuland et al. 2004; Seymour et al. 2008), while others do not. This is certainly to be expected for a population that marks the transition from an actively star-forming to a passively evolving evolutionary stage. A detailed study of the multiphase gas content of HzRGs for a more representative sample will be crucial to assess this question directly.

\subsection{Ionizing mechanism}

Closely related is the question which mechanism dominates the ionization of the gas? This is particularly important since our gas mass estimates (similar to those of Baum \& McCarthy 2000) do assume that most of the gas is photoionized, and in fact, the bolometric energy output of HzRGs seems sufficient to postulate that the nebulae may be matter bounded (Nesvadba et al. 2006b). Photoionization is also favoured by the measured UV line ratios (Villar-Martín et al. 1999). However, the gas kinematics suggests that shocks play a dominant role for the overall energy budget of the outflows, and hence it would appear natural that shocks would be an important ionizing source (a well-known puzzle, for more detailed discussions see e.g. Clark et al. 1998; Villar-Martin et al. 1997; Allen et al. 2008). Using the models of Dopita \& Sutherland $(1995,1996)$ for the line emission of fast shocks, we find that by assuming that shock ionization dominates, gas mass estimates would be about an order of magnitude larger than what we estimated from case B recombination (see Nesvadba et al. 2006b, for details). In this sense, and in spite of uncertainties of factors of a few, our gas mass estimates are rather conservative.

Ultimately, photoionization may explain why we observe larger amounts of ionized gas by several orders of magnitude than what is found in low-redshift radio galaxies, where fast, AGN-triggered outflows are predominantly seen in neutral gas through broad, blueshifted $\left(\sim 1000 \mathrm{~km} \mathrm{~s}^{-1}\right)$ HI absorption lines (Morganti et al. 2005). This dichotomy may be mainly due to the different radiative properties of the AGN rather than fundamentally different acceleration mechanisms for the outflows; HzRGs are harboring luminous, obscured quasars, and are embedded in large quantities of cold gas, whereas low-redshift radio galaxies seem to be radiatively inefficient and reside in hot, X-ray emitting halos. If this is the case, then we may significantly underestimate the frequency of AGN driven outflows, simply because they are observationally more subtle (see Morganti et al. 2005, for a more detailed discussion).

\subsection{Entrainment rates}

The amount of ionized gas and the dynamical time scales provide a rough estimate of the entrainment rate, as the cocoon plasma interacts with the ambient medium. The efficiency of 
the overpressurized plasma in entraining colder $\left(T \leq 10^{4} \mathrm{~K}\right)$ material is one of the crucial parameters in characterizing the efficiency by which the radio jet interacts with the ISM of a galaxy, and is a parameter that is now being addressed by detailed hydrodynamic simulations (e.g., Krause 2005; Sutherland $\&$ Bicknell 2007). However, since the amount of entrained material will strongly depend on the "macro" and "micro"-physics of the jet and ambient material, including several orders of magnitude in pressure, density, and size scales, it is difficult to derive this quantity from first principles.

The ionized gas we see will most likely be on the surface of filaments with low filling factors (Nesvadba et al. 2006b), that are being swept up and accelerated by the hot medium surrounding the radio jet. We can therefore give a purely empirical estimate of the entrainment rate, assuming that it roughly corresponds to the outflow rate of ionized gas, $M_{\text {ion }}$, and the dynamical timescale, $\tau$.

Recasting the discussion in Nesvadba et al. (2007a), we approximate the rate of entrained material, $M_{\mathrm{entr}, 9}$ (in multiples of $10^{9} M_{\odot}$ ), from the ionized gas masses of $\times 10^{10} M_{\odot}$ and dynamical timescales of few $\times 10^{7}$ yrs. This yields a simple scaling, $M_{\text {entr }, 9}=f_{\mathrm{c}} f_{\rho_{\mathrm{r}}} f_{\text {geom }} \dot{M}_{\text {ion }} \tau_{7}$, where $\tau_{7}$ indicates the dynamical time scale in units of $10^{7}$ yrs. $f_{\mathrm{c}}, f_{\rho_{\mathrm{r}}}$, and $f_{\text {geom }}$ parametrize our ignorance of the jet advance speed, radial density profile, and geometry, respectively. If we assume that all are of order unity for the relevant radii and time scales, we find an average entrainment rate of $\dot{M}_{\text {entr }} \sim 10^{2-3} M_{\odot} \mathrm{yr}^{-1}$. The cylindrical shape of the outflows suggests that the outflows do cover large solid angles, since they are related mainly to the expansion of the cocoon, and contrary to what may be expected from the pencil-like morphology of the jet.

The large ratio of ionized to molecular gas of order unity will make this estimate relatively robust. Nonetheless it will be a lower limit, given that we have no direct constraints on the amount of hot gas in the cocoon, and $\mathrm{CO}$ only traces the cold molecular gas with temperatures of order $\sim 10-100 \mathrm{~K}$. Krause (2005) argue that the mass of X-ray emitting gas within the cocoon may not greatly exceed the mass estimates for the colder gas given above. Carilli et al. (2002) estimate that the X-ray emission of MRC1138-262 may originate from up to $\sim 10^{12} M_{\odot}$ of hot gas, however, more recent X-ray studies of HzRGs favor the view that much of the emission originates from Compton scattering of photons from the cosmic microwave background on the relativistic electrons of the jet (e.g. Overzier et al. 2005), and accrodingly less X-ray emitting gas.

\subsection{Coupling efficiencies}

If jets are to cause the observed outflows, then they must provide at least the kinetic energy observed in the gas. The synchrotron emissivity of a radio source is a strong function of the ambient medium into which a jet expands. It is therefore difficult to estimate the intrinsic kinetic energy of the relativistic plasma from the luminosity of the radio source. Several methods have been proposed in the literature (e.g. Bicknell et al. 1997; Willott et al. 1999; Wan et al. 2000; Bîrzan et al. 2004; Kaiser \& Best 2007) which suggest that of order $0.1-10 \%$ of the kinetic energy of a jet is emitted in the radio. Similar to Nesvadba et al. (2006b) we adopt two methods to estimate the jet kinetic energy of the galaxies in our sample.

(1) A simple scaling, assuming that $10 \%$ of the kinetic energy is radiated at frequencies between $0.1-1 \mathrm{GHz}$ in the restframe.
Table 5. Radio properties of HzRGs.

\begin{tabular}{lcccc}
\hline \hline Source & $\begin{array}{c}D_{\text {ang }} \\
(1)\end{array}$ & $\begin{array}{c}D_{\text {phys }} \\
(3)\end{array}$ & $\begin{array}{c}\mathcal{L}_{0.1-1} \\
(4)\end{array}$ & $\begin{array}{c}\tau_{1.4 \mathrm{GHz}} \\
(5)\end{array}$ \\
\hline MRC0316-257 & 7.6 & 58 & 45.0 & 0.94 \\
MRC0406-244 & 7.3 & 59 & 45.2 & 0.96 \\
TXS0828+193 & 12.8 & 103 & 45.4 & 1.7 \\
\hline MRC1138-262 & 11.1 & 92 & 45.2 & 1. \\
\hline
\end{tabular}

For comparison we add MRC1138-262 from Nesvadba et al. (2006b). Column (1) - source name. Column (2) - maximal projected angular size of the radio source. Column (3) - maximal projected size in kpc. Column (4) - integrated luminosity between 0.1 and $1.0 \mathrm{GHz}$ in the restframe in $\mathrm{erg} \mathrm{s}^{-1} \mathrm{~cm}^{-2}$, corresponding to $\sim 0.001-0.1 \times$ the kinetic luminosity. Column (5) - age of the radio jet for a constant expansion speed of $0.01 c$ in $10^{7}$ yrs. Angular size distances are taken from De Breuck et al. (2001), radio luminosities are based on the VLA measurements of Carilli et al. (1997).

(2) The empirical relationship found by Wan et al. (2000) between the observed flux at $178 \mathrm{MHz}$ frequency and the kinetic radio power.

With the two methods, we find that the jets eject several $10^{46} \mathrm{erg} \mathrm{s}^{-1}$ in kinetic energy. Given that the adopted values are close to the lower bound of conversion factors from observed to intrinsic luminosities, these values serve effectively as lower bounds. For the estimates of individual galaxies see Table 5.

A critical quantity to gauge the overall impact of jet-driven AGN outflows is the efficiency with which the jet kinetic energy, $E_{\text {kin,jet }}$, is being translated into kinetic energy of the outflow, $E_{\text {kin,gas }}$, the coupling efficiency $\eta=E_{\text {kin,gas }} / E_{\text {kin,jet }}$. Obviously, such an estimate will not be very precise, given the large uncertainties in each measurement. Nonetheless, we feel that quantifying this value at order-of-magnitude precision will already be useful. With the values given in Table 6 , we find overall coupling efficiencies of $\sim 5-80 \%$.

Kaiser \& Alexander (1997) pointed out that for approximately adiabatic expansion, a radio source will become fainter as it expands. However, in our simple attempt to estimate jet kinetic energies, we did not take this into account. This may explain why we find the highest efficiencies in TXS0828+193, which is the largest source in our sample, by a factor $\sim 2$. We may simply underestimate the kinetic luminosity of the radio jet by factors of a few. We therefore favor a coupling efficiency of order $10 \%$. Similar efficiencies have been found in radio galaxies with outflows of neutral gas at significantly lower redshifts (Morganti et al. 2005), as well as in the X-ray cavities of massive lowredshift galaxy clusters (e.g. McNamara \& Nulsen 2007; Bîrzan et al. 2004; Rafferty et al. 2006). This may be a sign that the basic mechanism in all these environments may not be fundamentally different although they act on different scales.

\section{The global role of outflows in HzRGs}

One of the main questions we address in this study is on the frequency of highly turbulent emission line gas and energetic outflows in HzRGs, similar to what we observed in our SINFONI study of MRC1138-262. Such outflows may be an important mechanism in terminating the vigorous starbursts observed in galaxies near the upper end of the galaxy mass function during the "Quasar Era", if they are powerful enough to efficiently heat and unbind a significant fraction of the interstellar medium of a massive, gas-rich galaxy. The presence of such outflows in another three HzRGs with extended radio sources (among three 
Table 6. Outflow properties of HzRGs.

\begin{tabular}{lcccccc}
\hline \hline $\begin{array}{l}\text { Source } \\
(1)\end{array}$ & $\begin{array}{c}D_{\text {opt }} \\
(2)\end{array}$ & $\begin{array}{c}\Delta v \\
(3)\end{array}$ & $\begin{array}{c}\tau_{\text {opt }} \\
(4)\end{array}$ & $\begin{array}{c}\dot{E}_{\text {kin,obs }} \\
(5)\end{array}$ & $\begin{array}{c}\dot{E}_{\text {kin,ext }} \\
(6)\end{array}$ & $\begin{array}{c}\dot{E}_{\text {shell }} \\
(7)\end{array}$ \\
\hline MRC0316-257 & $21 \times 9$ & 670 & 3.1 & & & 44.4 \\
MRC0406-244 & $30 \times 9$ & 960 & 2.9 & 45.3 & 45.6 & 45.0 \\
TXS0828+193 & $28 \times 10$ & 800 & 4.1 & 45.3 & 45.3 & 45.0 \\
\hline MRC1138-262 & $53 \times 32$ & 800 & 1 & 44.2 & & 45.6 \\
\hline
\end{tabular}

For comparison we add MRC1138-262 from Nesvadba et al. (2006b). Column (1) - source name. Column (2) - extent of the optical line emission in kpc along the major and minor axis, respectively. Column (3) velocity offset between the blue and redshifted bubble in $\mathrm{km} \mathrm{s}^{-1}$ in the rest-frame. Column (4) - age of the outflow for constant $\Delta v$ and neglecting projection effects in $10^{7}$ yrs. Column (5) - energy injection necessary to power the outflow in erg s ${ }^{-1}$ ("method 1", see Sect. 5.5 for details), and mass estimates based on the measured $\mathrm{H} \alpha$ fluxes, uncorrected for extinction. This serves as a strict lower limit to the kinetic energy injection. Column (6) - the same as Col. (5), but using extinctioncorrected $\mathrm{H} \alpha$ fluxes. Column (7) - energy injection necessary to inflate a hot bubble in erg s ${ }^{-1}$ ("method 2", see Sect. 5.5 for details).

Table 7. H $\alpha$ luminosities and ionized gas masses.

\begin{tabular}{|c|c|c|c|c|c|}
\hline $\begin{array}{l}\text { Source } \\
(1)\end{array}$ & $\begin{array}{c}F_{\mathrm{H} \alpha} \\
(2)\end{array}$ & $\begin{array}{c}F_{\mathrm{H} \alpha}^{\text {corr }} \\
(3)\end{array}$ & $\begin{array}{c}\mathcal{L}(\mathrm{H} \alpha) \\
(4)\end{array}$ & $\begin{array}{c}\mathcal{L}(\mathrm{H} \alpha)^{\text {corr }} \\
(5)\end{array}$ & $\begin{array}{c}\log \left(M_{\mathrm{H} \alpha}\right) \\
(6)\end{array}$ \\
\hline $0406-244$ & -14.1 & -13.4 & 44.5 & 45.2 & 10.6 \\
\hline $0828+193$ & -14.2 & -13.4 & 44.4 & 45.3 & 10.7 \\
\hline
\end{tabular}

Column (1) - source ID. Column (2) - measured integrated $\mathrm{H} \alpha$ flux in erg $\mathrm{s}^{-1} \mathrm{~cm}^{-2}$. Column (3) - extinction corrected $\mathrm{H} \alpha$ flux in erg $\mathrm{s}^{-1} \mathrm{~cm}^{-2}$. Column (4) - integrated measured $\mathrm{H} \alpha$ luminosity in erg s$~^{-1}$. Column (5) - integrated extinction corrected $\mathrm{H} \alpha$ luminosity in erg s$~^{-1}$. Column (6) - ionized gas mass estimate.

galaxies observed) suggest that AGN-driven outflows are a common phenomenon in $z \sim 2-3$ HzRGs.

Overall, the physical conditions and gas kinematics in MRC1138-262 and the three galaxies of the present sample are very similar. All four galaxies have typical line widths of $F W H M \sim 800-1000 \mathrm{~km} \mathrm{~s}^{-1}$ over large areas, suggesting turbulent energy injection rates of $\sim 10^{44} \mathrm{erg} \mathrm{s}^{-1}$. Areas with larger widths may be due to overlap of adjacent bubbles. $\mathrm{H} \alpha$ fluxes indicate fairly similar ionized gas masses in all four galaxies. The physical conditions within the outflows seem also largely similar, with electron densities of several hundred $\mathrm{cm}^{-3}$ for MRC0406-244 and MRC1138-262. Both TXS0828+193 and MRC0406-244 have [OIII] emission line ratios consistent with temperatures of $\sim 10^{4} \mathrm{~K}$. Relative velocities between individual bubbles are $\sim 1000 \mathrm{~km} \mathrm{~s}^{-1}$ in all cases, suggesting velocities near the escape velocity of a massive dark matter halo. Differences are mainly in the emission line morphology where MRC1138-262 seems particularly irregular. The reason may be different evolutionary stages or perhaps that MRC1138-262 has a more extreme environment. This is suggested by the particularly large velocity dispersion of companion galaxies (e.g., Venemans et al. 2007; Miley et al. 2006) and an exceptionally large rotation measure (Pentericci et al. 2000). However, we cannot rule out that the greater complexity of MRC1138-262 is simply the result of different orientations, with the AGN of MRC1138-262 being less inclined relative to the line of sight. MRC1138-262 hosts an obscured quasar evidenced by broad nuclear $\mathrm{H} \alpha$ line emission (Nesvadba et al. 2006b).

The global dynamical properties of the emission line gas however do appear very similar in all 4 galaxies of our current sample, in spite of this range in the appearance of individual sources. This suggests that powerful outflows in HzRGs likely are a common phenomenon. Studying the outflows in a larger sample of HzRGs with and without broad $\mathrm{H} \alpha$ lines may help resolving this issue, and we have now started observations of a significantly larger sample of $\sim 30 \mathrm{HzRG}$ with SINFONI.

\subsection{Subsequent mass assembly of HzRGs}

The properties of HzRGs suggest that they are near the end of their main epoch of active star-formation, as seen, e.g., from the evolution towards very regular, probably elliptical continuum profiles in HzRGs at $z \sim 2$ (e.g., van Breugel et al. 1998). This is certainly consistent with postulating that the AGN winds we observe are truncating the star-formation epoch in the host galaxy. However, will the fingerprints of this epoch be preserved until $z=0$ ? HzRGs reside in overdense environments, and may double their stellar mass through subsequent satellite accretion (as predicted by galaxy evolution models, De Lucia et al. 2006).

We can roughly estimate the accretion-dominated growth of HzRGs from the number of companion galaxies observed around many HzRGs, including MRC0316-257 and MRC1138262 (e.g. Le Fevre et al. 1996; Venemans et al. 2007, 2005; Kurk et al. 2004; Best et al. 2003). Continuum luminosities and $\mathrm{H} \alpha$ line widths (e.g., Kurk et al. 2004) imply masses of typically few $\times 10^{9} M_{\odot}$. With up to $\sim 50$ companions, the central galaxy may gain of order $10^{11} M_{\odot}$, if all companions are accreted, corresponding to about half its stellar mass. For gas fractions of order 50\%, satellite accretion may fuel the formation of $\sim 20-30 \%$ of the total stellar mass in the descendent of a HzRG at $z \sim 0$.

However, this will not inevitably dilute the fingerprints of previous AGN feedback, such as the low content of cold and warm gas or truncated star-formation histories of massive galaxies at low redshift (e.g., Pipino \& Matteucci 2004). Dynamical interactions in the dense environment of HzRGs will lead to premature ageing of the stellar population in the satellites and efficient gas removal, similar to low-redshift galaxy clusters. Boselli et al. (2008) find for dwarf galaxies in the Virgo cluster, that ram pressure stripping alone may turn strongly star-forming irregular galaxies into gas-poor ellipticals within 1-2 crossing times (few 100 Myr for a HzRG halo) and lift the satellite onto the red sequence. (Interestingly, the red sequence seems to fall in place at $z \sim 2$, as shown Kodama et al. 2007, for the overdensities surrounding MRC1138-262 and MRC0316-257.) The metal enrichment of the ISM will be particularly efficient, resulting in a metallicity increase of about 0.5 dex relative to the field (Boselli et al. 2008). Comparing with the mass-metallicity relationship of Tremonti et al. (2004) we find that this will compensate for the metallicity difference between a $5 \times 10^{9} M_{\odot}$ galaxy and a $10^{11} M_{\odot}$ galaxy.

AGN outflows may enhance these processes, either through gas stripping when a satellite crosses the outflow (Irwin et al. 1987; Fujita 2008), or by increasing the entropy and pressure in the surrounding hot medium (Nath \& Roychowdhury 2002; McCarthy et al. 2008), where an AGN-driven pre-heating phase at $z \sim 1-3$ appears sufficient to explain the observed temperature profiles of massive galaxy clusters. In either case, the satellite galaxies will likely undergo "dry" mergers with low dissipation, and may have a surprisingly small impact on the properties of the low-redshift descendents of HzRGs. Dry merging may also explain why HzRGs seem to have smaller effective radii than massive spheroidal galaxies at low redshift, as well as the extended stellar envelopes of cD galaxies (Schombert 1987). 


\subsection{The cosmological significance of jet-driven outflows}

With only $6 \mathrm{HzRG}$ with rest-frame optical IFU data (including targets from Nesvadba et al. 2006, 2007b), our sample is relatively small. However, the great similarity of the ionized gas masses and kinetic properties is intriguing, and raises confidence that similar outflows are common among powerful radio galaxies during the era of luminous quasars. Moreover, large ionized gas masses, line widths, and velocity gradients have been reported previously, based on observations with longslit spectrographs of radio galaxies at redshifts $z \sim 1$ and in a few cases below (e.g. Baum \& McCarthy 2000; Villar-Martín et al. 2002, 2003, 1999; McCarthy et al. 1996; Tadhunter 1991; Inskip et al. 2002; Clark et al. 1998; Best et al. 1997).

In Sect. 7.4 we argued that of order $10 \%$ of the jet kinetic energy is transformed into kinetic energy of the gas. We will in the following assume that this is a typical value for powerful radio sources at $z \sim 2$. We can then use the observed radio luminosity function of steep-spectrum radio sources to estimate the global energy release of jet-driven AGN feedback into the intergalactic medium at high redshift.

We use the radio luminosity function of Willott et al. (2001), who find that the radio-loud population can be split into two classes, a low-luminosity population associated with galaxies with low-luminosity emission lines including FRI and FRII radio structures, and a high-luminosity population consisting of radio galaxies and quasars with strong emission lines, and with nearly exclusively FRII radio sources. We only include this luminous population into our estimates, and conservatively use their set of model parameters which minimizes our estimate. The comoving number density of this population rises by nearly 3 orders of magnitude between $z=0$ and $z=2$, and has properties that generally agree very well with the sources we observe.

We computed the luminosity function for redshifts $z=$ $0-4$ in bins of $z=0.1$, and use the peak of the co-moving number density at a radio-power at $151 \mathrm{MHz}$ of $\mathcal{L}_{151}=$ $10^{27} \mathrm{~W} \mathrm{~Hz}^{-1} \mathrm{sr}^{-1}$, which is sufficiently close to the radio power of the sources in our sample. We then compute the co-moving number density per redshift bin, $\Phi_{\text {mod }}$, converting to our cosmology. Since the radio-loud phase, $\tau_{\text {jet }}$ is significantly shorter than the cosmic time within these bins, we estimate co-moving number densities corrected for the duty cycle, $\Phi_{\mathrm{rl}, \mathrm{c}}$, i.e. $\Phi_{\mathrm{rl}, \mathrm{c}}=$ $\frac{t_{\text {bin }}}{\tau_{\text {jet }}} \Phi_{\text {mod }}$, where $t_{\text {bin }}$ indicates the cosmic time elapsed within each redshift bin, and $\tau_{\text {jet }}$ indicates the lifetime of the radio jet. We find a global energy release at redshifts $z=1-3$ (i.e., near the "Quasar Era"), $\mathrm{d} \rho_{\mathrm{E}, \mathrm{kin}} / \mathrm{d} t$

$\mathrm{d} \rho_{\text {E,kin }} / \mathrm{d} t=9 \times 10^{56} \tau_{7}^{-1} \mathrm{~d} E_{\text {kin } 60} / \mathrm{d}_{60} \operatorname{erg~s}^{-1} \mathrm{Mpc}^{-3}$,

where $\tau_{7}$ is given in $10^{7} \mathrm{yrs}$, and $\mathrm{d} E_{\mathrm{kin}} / \mathrm{d} t_{60}$ is given in $10^{60} \mathrm{erg}$. For the redshift evolution of Willott et al. (2001) this corresponds to about $90 \%$ of the energy release at all redshifts, emitted during $\sim 3.6$ Gyr.

\subsection{Overall feedback efficiency}

To quantify the overall efficiency of AGN feedback as observed in powerful radio galaxies, we will now compare the observed kinetic energy injection of the ionized gas within an activity phase with the possible total energy release through accretion onto the supermassive black hole. This simply corresponds to the relationship between the total energy equivalent of the rest mass of a black hole of a given mass and the kinetic energy of the outflows,

$\eta=5.6 \times 10^{-2} E_{\mathrm{kin}, 60} t_{7} M_{9}^{-1}$

$E_{\text {kin,60 }}$ indicates the energy injection in multiples of $10^{60} \mathrm{erg}$, $t_{7}$ the phase of AGN activity in multiples of $10^{7} \mathrm{yrs}$, and $M_{\mathrm{BH}, 9}$ the mass of the supermassive black hole in multiples of $10^{9} M_{\odot}$. The efficiency $\eta$ is given in percent.

Black hole masses for HzRGs are not easily derived directly. For an order-of-mass estimate, we will assume that the low-redshift relationship between black hole and bulge mass will approximately hold (Magorrian et al. 1998; Ferrarese 2002; Tremaine et al. 2002; Häring \& Rix 2004). The light profiles of HzRGs at $z \sim 2$ are regular and likely elliptical (van Breugel et al. 1998; Pentericci et al. 1999), suggesting that these are bulge-dominated systems. Adopting the stellar mass estimates of Seymour et al. (2007) for HzRGs, $\log M_{\text {bulge }} \sim 11-11.5$, and the relationship of Häring \& Rix (2004), black hole masses are about $\log M_{\mathrm{bh}} \sim 8.2-8.8$. This implies that of order $0.1 \%$ of the energy equivalent of the black holes in HzRGs are released in kinetic energy of the outflows. This is a purely empirical estimate, and does not rely on any assumption regarding the efficiency of accretion or feedback.

To investigate this question more globally, we can also relate the global energy density released by powerful radio sources derived in Sect. 8.2, $\rho_{\text {obs }} \sim 9 \times 10^{56} \mathrm{erg} \mathrm{Mpc}^{-3}$, with the local mass density of black holes, estimated by Yu \& Tremaine (2002), $\rho_{\mathrm{BH}}=2.9 \pm 0.5 \times 10^{5} h_{70}^{2} M_{\odot} \mathrm{Mpc}^{-3}$, corresponding to a total energy equivalent of $\rho_{\mathrm{e}, \mathrm{kin}} \sim 5.2 \times 10^{59} \mathrm{erg} \mathrm{Mpc}^{-3}$. This will serve as a strict lower limit on the efficiency with which the rest-frame energy equivalent of the black hole mass has been translated into outflow energy in the past. We find an efficiency of about $0.2 \%$ with which the rest mass of the black hole is being translated into outflow energy, in agreement with the above estimate based on the masses of supermassive black holes in HzRGs, and with recent estimates comparing the optical and radio luminosity function of radio-loud AGN, but without the direct observations of AGN-driven winds (Shankar et al. 2008).

The efficiency of AGN feedback in terms of the black hole accretion is an important parameter in cosmological models of galaxy evolution and structure formation, which typically adopt a factor of $\sim 0.5 \%$ (e.g. Scannapieco \& Oh 2004; Springel et al. 2005; Di Matteo et al. 2005; Chakrabarti et al. 2007). Given the uncertainties of factors of a few, and the fact that our estimates are based on the ionized gas only, the agreement is remarkable. This underlines the potentially significant role of jet-driven outflows for galaxy evolution.

\section{Summary and conclusions}

We studied the kinematics and physical characteristics of the extended emission line regions in 3 powerful radio galaxies at $z \sim 2$, using integral-field spectroscopy of the rest-frame optical emission lines. Our results are consistent with what is expected from giant, jet-driven AGN outflows, similar to what we found in an earlier study of the $z \sim 2.2$ galaxy MRC1138262 (Nesvadba et al. 2006b). Analysis of the emission and continuum line morphologies, velocity maps, and flow orientation relative to the orientation of the radio jet does not favor alternative interpretations, e.g., merging, rotation, or gas infall. Such outflows may be common in powerful high-redshift radio galaxies (HzRGs) during the "Quasar Era". Overall, we 
find energetic outflows in all $4 z \sim 2$ powerful radio galaxies with extended jets for which we obtained rest-frame optical integral-field spectroscopy. Comparison with previous longslit spectroscopy (Baum \& McCarthy 2000) suggests similar properties in $>10$ HzRGs at $z>1$.

The emission line regions extend over $\sim 10 \mathrm{kpc} \times 30 \mathrm{kpc}$, and are elongated along the axis of the radio jets. Most of the complex morphologies of these galaxies, previously observed with broadband imaging, appears to be due to line emission. The line-free continuum emission extracted from the data cube is relatively faint and compact $(\leq 10 \mathrm{kpc})$. Assuming simple case-B recombination, we find of order $10^{10} M_{\odot}$ of ionized gas, similar to the typical cold molecular gas content of HzRGs traced by CO line emission. From observed line ratios, we can constrain the physical properties of the gas. We detect [OIII] $\lambda 4363$ in a $z \sim 2$ galaxy, allowing for a direct estimate of the electron temperature of $\sim 10^{4} \mathrm{~K}$. Electron densities are derived from the $[\mathrm{SII}] \lambda \lambda 6716,6731$ line ratio and are $\sim 500 \mathrm{~cm}^{-3}$. From the measured $\mathrm{H} \alpha / \mathrm{H} \beta$ line ratio we estimate extinction of $A(\mathrm{H} \beta) \sim 1-4$ mag.

The velocity fields are very similar in all 3 sources and are reminiscent of two bubbles expanding back-to-back from the AGN. Velocity offsets near the center of the host galaxy are large, 700-1000 $\mathrm{km} \mathrm{s}^{-1}$, but velocities within each bubble are relatively uniform. Line widths are of order FWHM $1000 \mathrm{~km} \mathrm{~s}^{-1}$, indicating strong turbulence. Larger widths in the central regions are likely due to overlaps between the bubbles. Comparison with the orientation of the radio jets suggest that this gas is outflowing.

With kinetic energies of order $10^{60}$ erg required to power the observed emission line kinematics, these outflows may be sufficient to remove most of the interstellar medium of the host galaxies in a nearly explosive event. Observed kinetic energies correspond to $\sim 0.2 \%$ of the energy equivalent of the rest mass of the supermassive black holes, in good agreement with AGN feedback prescriptions used in galaxy evolution models

The following observations lead us to argue that the outflows are dominated by the radio jets: (1) geometry: good alignment with the radio jets and elongation along the jet axis. The gas is blueshifted on the side of the approaching radio jet; (2) size: the sizes of the emission line regions do not reach beyond the radio lobes. Nesvadba et al. (2007a) do not find extended outflows in galaxies with compact radio sources observed with SINFONI. Emission line gas outside the radio lobes observed in Ly $\alpha$ is kinematically quiescent (Villar-Martín et al. 2003); (3) timescales: dynamical timescales of the outflows are few $\times 10^{7} \mathrm{yrs}$, similar to typical ages of radio jets; (4) energies: kinetic energies of the outflows correspond to about $10 \%$ of the jet kinetic energy.

Comparing with the $z \sim 2$ radio luminosity function of Willott et al. (2001), we find that AGN winds like those observed may release about $9 \times 10^{56} \mathrm{erg} \mathrm{s}^{-1} \mathrm{Mpc}^{-3}$ in energy density from the massive host galaxies of powerful radio sources at $z \sim 2$. This energy may have a long-term impact on the subsequent evolution of the AGN host galaxy as well as nearby galaxies and help maintaining the "anti-hierarchical" properties of massive galaxies in spite of possible subsequent merging with companion galaxies. Following Nath \& Roychowdhury (2002); McCarthy et al. (2008), much of this energy may contribute to increasing the entropy and pressure in the environment of massive early type galaxies and clusters, and enhance the efficiency of dynamical interactions with galaxies in the environment of HzRGs, such as ram pressure stripping. Comparing with studies of ram-pressure stripping (Boselli et al. 2008) we find that the satellite galaxies may already be red, poor in gas and rich in metals when they are being accreted onto the central galaxy. In spite of subsequent growth by up to $\sim 50 \%$ in stellar mass, we find that minor "dry" mergers with ram-pressure stripped satellites would not strongly influence the observed evolutionary properties of the central galaxy.

Acknowledgements. The authors wish to thank C. Carilli for interesting discussions with and without good Bavarian beer, and for generously sharing his VLA imaging. N.P.H.N. is grateful to R. Daly, C. Kaiser, A. Pippino, J. Ostriker and M. Villar-Martin for interesting and inspiring discussions. She also wishes to thank M. Krause and V. Gaibler for sharing their insight into hydrodynamical modeling of radio jets and their hospitality at the Landessternwarte in Heidelberg. We thank D. Downes for valuable discussions and a particularly careful reading of the paper. Comments by the anonymous referee helped improve the paper. We thank the ESO OPC for their allocation of observing time and the staff at Paranal for their help and support in making these observations. N.P.H.N. wishes to acknowledge financial support from the European Commission through a Marie Curie Postdoctoral Fellowship and M.D.L. wishes to thank the Centre National de la Recherche Scientifique for its continuing support of his research. W.v.B. acknowledges support for radio galaxy studies at UC Merced, including the work reported here, with the Hubble Space Telescope and the Spitzer Space Telescope via NASA grants HST \#10127, SST \#1264353, SST \#1265551, SST \#1279182 and SST \#1281587.

\section{References}

Alexander, P., \& Leahy, J. P. 1987, MNRAS, 225, 1

Alexander, P., \& Pooley, G. G. 1996, The large-scale structure, dynamics and thermodynamics of Cygnus A, Cygnus A - Studay of a Radio Galaxy, 149

Allen, M. G., Groves, B. A., Dopita, M. A., Sutherland, R. S., \& Kewley, L. J. 2008, ArXiv e-prints

Archibald, E. N., Dunlop, J. S., Hughes, D. H., et al. 2001, MNRAS, 323, 417

Armus, L., Soifer, B. T., Murphy, Jr., T. W., et al. 1998, ApJ, 495, 276

Baum, S. A., \& McCarthy, P. J. 2000, AJ, 119, 2634

Best, P. N., Longair, M. S., \& Roettgering, J. H. A. 1997, MNRAS, 292, 758

Best, P. N., Lehnert, M. D., Miley, G. K., \& Röttgering, H. J. A. 2003, MNRAS, 343, 1

Best, P. N., Kauffmann, G., Heckman, T. M., et al. 2005, MNRAS, 362, 25

Best, P. N., Kaiser, C. R., Heckman, T. M., \& Kauffmann, G. 2006, MNRAS, 368, L67

Bicknell, G. V., Dopita, M. A., \& O’Dea, C. P. O. 1997, ApJ, 485, 112

Binney, J., \& Tabor, G. 1995, MNRAS, 276, 663

Bîrzan, L., Rafferty, D. A., McNamara, B. R., Wise, M. W., \& Nulsen, P. E. J. 2004, ApJ, 607, 800

Blundell, K. M., \& Rawlings, S. 1999, Nature, 399, 330

Boehringer, H., Voges, W., Fabian, A. C., Edge, A. C., \& Neumann, D. M. 1993,

MNRAS, 264, L25

Bonnet, H., Abuter, R., Baker, A., et al. 2004, The Messenger, 117, 17

Boselli, A., Boissier, S., Cortese, L., \& Gavazzi, G. 2008, ApJ, 674, 742

Bournaud, F., Daddi, E., Elmegreen, B. G., et al. 2008, ArXiv e-prints

Bower, R. G., Benson, A. J., Malbon, R., et al. 2006, MNRAS, 370, 645

Capetti, A., Axon, D. J., Macchetto, F. D., Marconi, A., \& Winge, C. 1999, ApJ, 516,187

Carilli, C. L., Roettgering, H. J. A., van Ojik, R., Miley, G. K., \& van Breugel, W. J. M. 1997, ApJS, 109, 1

Carilli, C. L., Harris, D. E., Pentericci, L., et al. 2002, ApJ, 567, 781

Chakrabarti, S., Cox, T. J., Hernquist, L., et al. 2007, ApJ, 658, 840

Ciotti, L., \& Ostriker, J. P. 2007, ApJ, 665, 1038

Clark, N. E., Axon, D. J., Tadhunter, C. N., Robinson, A., \& O’Brien, P. 1998, ApJ, 494, 546

Croton, D. J., Springel, V., White, S. D. M., et al. 2006, MNRAS, 365, 11

De Breuck, C., Röttgering, H., Miley, G., van Breugel, W., \& Best, P. 2000, A\&A, 362, 519

De Breuck, C., van Breugel, W., Röttgering, H., et al. 2001, AJ, 121, 1241

De Breuck, C., Neri, R., \& Omont, A. 2003, New Astron. Rev., 47, 285

De Breuck, C., Downes, D., Neri, R., et al. 2005, A\&A, 430, L1

De Lucia, G., Springel, V., White, S. D. M., Croton, D., \& Kauffmann, G. 2006, MNRAS, 366, 499

De Young, D. S. 1991, ApJ, 371, 69

Di Matteo, T., Springel, V., \& Hernquist, L. 2005, Nature, 433, 604

Donoso, E., Best, P. N., \& Kauffmann, G. 2008, [arXiv. 0809. 2076]

Dopita, M. A., \& Sutherland, R. S. 1995, ApJ, 455, 468

Dopita, M. A., \& Sutherland, R. S. 1996, ApJS, 102, 161

Dyson, J. E., \& Williams, D. A. 1980, Physics of the interstellar medium (New York: Halsted Press), 204 
Erb, D. K., Steidel, C. C., Shapley, A. E., et al. 2006, ApJ, 647, 128

Evans, A. S. 1998, ApJ, 498, 553

Ferrarese, L. 2002, ApJ, 578, 90

Förster Schreiber, N. M., Genzel, R., Lehnert, M. D., et al. 2006, ApJ, 645, 1062 Fragile, P. C., Murray, S. D., Anninos, P., \& van Breugel, W. 2004, ApJ, 604, 74

Fujita, Y. 2008, MNRAS, 384, L41

Gaibler, V., Camenzind, M., \& Krause, M. 2007, ArXiv e-prints, 710

Garrington, S. T., Leahy, J. P., Conway, R. G., \& Laing, R. A. 1988, Nature, 331, 147

Genzel, R., Tacconi, L. J., Eisenhauer, F., et al. 2006, Nature, 442, 786

Greve, T. R., Bertoldi, F., Smail, I., et al. 2005, MNRAS, 359, 1165

Häring, N., \& Rix, H.-W. 2004, ApJ, 604, L89

Heckman, T. M., Lehnert, M. D., Miley, G. K., \& van Breugel, W. 1991a, ApJ, 381,373

Heckman, T. M., Miley, G. K., Lehnert, M. D., \& van Breugel, W. 1991b, ApJ, 370, 78

Hopkins, P. F., Hernquist, L., Cox, T. J., et al. 2006, ApJS, 163, 1

Humphrey, A., Villar-Martín, M., Vernet, J., et al. 2008, MNRAS, 383, 11

Inskip, K. J., Best, P. N., Rawlings, S., et al. 2002, MNRAS, 337, 1381

Irwin, J. A., Seaquist, E. R., Taylor, A. R., \& Duric, N. 1987, ApJ, 313, L91

Iwamuro, F., Motohara, K., Maihara, T., et al. 2003, ApJ, 598, 178

Kaiser, C. R., \& Alexander, P. 1997, MNRAS, 286, 215

Kaiser, C. R., \& Best, P. N. 2007, MNRAS, 381, 1548

Kaiser, C. R., \& Binney, J. 2003, MNRAS, 338, 837

Kaiser, C. R., Dennett-Thorpe, J., \& Alexander, P. 1997a, MNRAS, 292, 723

Kaiser, C. R., Dennett-Thorpe, J., \& Alexander, P. 1997b, MNRAS, 292, 723

King, A. 2003, ApJ, 596, L27

Klamer, I. J., Ekers, R. D., Sadler, E. M., et al. 2005, ApJ, 621, L1

Kodama, T., Tanaka, I., Kajisawa, M., et al. 2007, MNRAS, 377, 1717

Krause, M. 2005, A\&A, 431, 45

Krause, M., \& Alexander, P. 2007, MNRAS, 376, 465

Kronberger, T., Kapferer, W., Schindler, S., \& Ziegler, B. L. 2007, A\&A, 473, 761

Kurk, J. D., Pentericci, L., Overzier, R. A., Röttgering, H. J. A., \& Miley, G. K. 2004, A\&A, 428, 817

Laing, R. A. 1988, Nature, 331, 149

Law, D. R., Steidel, C. C., Erb, D. K., et al. 2007, ApJ, 669, 929

Le Fevre, O., Deltorn, J. M., Crampton, D., \& Dickinson, M. 1996, ApJ, 471, L11

Magorrian, J., Tremaine, S., Richstone, D., et al. 1998, AJ, 115, 2285

McCarthy, P. J., van Breughel, W., Kapahi, V. K., \& Subrahmanya, C. R. 1991, AJ, 102, 522

McCarthy, P. J., Elston, R., \& Eisenhardt, P. 1992, ApJ, 387, L29

McCarthy, P. J., Baum, S. A., \& Spinrad, H. 1996, ApJS, 106, 281

McCarthy, I. G., Babul, A., Bower, R. G., \& Balogh, M. L. 2008, MNRAS, 403

McNamara, B. R., \& Nulsen, P. E. J. 2007, ARA\&A, 45, 117

Mellema, G., Kurk, J. D., \& Röttgering, H. J. A. 2002, A\&A, 395, L13

Miley, G. K., Overzier, R. A., Zirm, A. W., et al. 2006, ApJ, 650, L29

Morganti, R., Tadhunter, C. N., \& Oosterloo, T. A. 2005, A\&A, 444, L9

Murgia, M., Fanti, C., Fanti, R., et al. 1999, A\&A, 345, 769

Murray, N., Quataert, E., \& Thompson, T. A. 2005, ApJ, 618, 569

Nath, B. B., \& Roychowdhury, S. 2002, MNRAS, 333, 145

Neri, R., Genzel, R., Ivison, R. J., et al. 2003, ApJ, 597, L113

Nesvadba, N. P. H., Lehnert, M. D., Eisenhauer, F., et al. 2006a, ApJ, 650, 661

Nesvadba, N. P. H., Lehnert, M. D., Eisenhauer, F., et al. 2006b, ApJ, 650, 693

Nesvadba, N. P. H., Lehnert, M. D., De Breuck, C., Gilbert, A., \& van Breugel, W. 2007a, A\&A, 475, 145

Nesvadba, N. P. H., Lehnert, M. D., Genzel, R., et al. 2007b, ApJ, 657, 725

Nesvadba, N. P. H., Lehnert, M. D., Davies, R. I., Verma, A., \& Eisenhauer, F. 2008, A\&A, 479, 67
Overzier, R. A., Harris, D. E. Carilli, C. L., et al. 2005, A\&A, 433, 87

Owsianik, I., \& Conway, J. E. 1998, A\&A, 337, 69

Papadopoulos, P. P., Röttgering, H. J. A., van der Werf, P. P., et al. 2000, ApJ, 528, 626

Pentericci, L., Röttgering, H. J. A., Miley, G. K., et al. 1999, A\&A, 341, 329

Pentericci, L., Van Reeven, W., Carilli, C. L., Röttgering, H. J. A., \& Miley, G. K. 2000, A\&AS, 145, 121

Pipino, A., \& Matteucci, F. 2004, MNRAS, 347, 968

Pipino, A., Matteucci, F., \& D'Ercole, A. 2007, ArXiv e-prints

Rafferty, D. A., McNamara, B. R., Nulsen, P. E. J., \& Wise, M. W. 2006, ApJ, 652,216

Reuland, M., Röttgering, H., van Breugel, W., \& De Breuck, C. 2004, MNRAS, 353,377

Rocca-Volmerange, B., Le Borgne, D., De Breuck, C., Fioc, M., \& Moy, E. 2004, A\&A, 415, 931

Rudnick, G., Rix, H.-W., Franx, M., et al. 2003, ApJ, 599, 847

Rush, B., McCarthy, P. J., Athreya, R. M., \& Persson, S. E. 1997, ApJ, 484, 163 Ruszkowski, M., \& Begelman, M. C. 2002, ApJ, 581, 223

Saxton, C. J., Bicknell, G. V., Sutherland, R. S., \& Midgley, S. 2005, MNRAS, 359,781

Sazonov, S. Y., Ostriker, J. P., Ciotti, L., \& Sunyaev, R. A. 2005, MNRAS, 358, 168

Scannapieco, E., \& Oh, S. P. 2004, ApJ, 608, 62

Schombert, J. M. 1987, ApJS, 64, 643

Seymour, N., Stern, D., De Breuck, C., et al. 2007, ApJS, 171, 353

Seymour, N., Ogle, P., De Breuck, C., et al. 2008, ApJ, 681, L1

Shankar, F., Cavaliere, A., Cirasuolo, M., \& Maraschi, L. 2008, ApJ, 676, 131

Silk, J., \& Rees, M. J. 1998, A\&A, 331, L1

Smail, I., Ivison, R. J., Blain, A. W., \& Kneib, J.-P. 2002, MNRAS, 331, 495

Springel, V., Di Matteo, T., \& Hernquist, L. 2005, MNRAS, 361, 776

Steidel, C. C., Giavalisco, M., Pettini, M., Dickinson, M., \& Adelberger, K. L. 1996, ApJ, 462, L17

Sutherland, R. S., \& Bicknell, G. V. 2007, ApJS, 173, 37

Swinbank, A. M., Chapman, S. C., Smail, I., et al. 2006, MNRAS, 371, 465

Tadhunter, C. N. 1991, MNRAS, 251, 46P

Tody, D. 1993, in Astronomical Data Analysis Software and Systems II, ed. R. J. Hanisch, R. J. V. Brissenden, \& J. Barnes, ASP Conf. Ser., 52, 173

Tremaine, S., Gebhardt, K., Bender, R., et al. 2002, ApJ, 574, 740

Tremonti, C. A., Heckman, T. M., Kauffmann, G., et al. 2004, ApJ, 613, 898 van Breugel, W. J. M., Stanford, S. A., Spinrad, H., Stern, D., \& Graham, J. R. 1998, ApJ, 502, 614

Venemans, B. P., Röttgering, H. J. A., Miley, G. K., et al. 2005, A\&A, 431, 793

Venemans, B. P., Röttgering, H. J. A., Miley, G. K., et al. 2007, A\&A, 461, 823

Villar-Martin, M., Tadhunter, C., \& Clark, N. 1997, A\&A, 323, 21

Villar-Martín, M., Tadhunter, C., Morganti, R., Axon, D., \& Koekemoer, A. 1999, MNRAS, 307, 24

Villar-Martín, M., Vernet, J., di Serego Alighieri, S., et al. 2002, MNRAS, 336, 436

Villar-Martín, M., Vernet, J., di Serego Alighieri, S., et al. 2003, MNRAS, 346, 273

Villar-Martín, M., Sánchez, S. F., De Breuck, C., et al. 2006, MNRAS, 366, L1 Villar-Martín, M., Sánchez, S. F., Humphrey, A., et al. 2007, MNRAS, 378, 416 Wan, L., Daly, R. A., \& Guerra, E. J. 2000, ApJ, 544, 671

Willott, C. J., Rawlings, S., Blundell, K. M., \& Lacy, M. 1999, MNRAS, 309, 1017

Willott, C. J., Rawlings, S., Blundell, K. M., Lacy, M., \& Eales, S. A. 2001, MNRAS, 322, 536

Willott, C. J., Rawlings, S., Jarvis, M. J., \& Blundell, K. M. 2003, MNRAS, 339, 173

Wright, S. A., Larkin, J. E., Barczys, M., et al. 2007, ApJ, 658, 78

Yu, Q., \& Tremaine, S. 2002, MNRAS, 335, 965 